\title{
QUOTIENTS OF BOUNDED NATURAL FUNCTORS
}

\author{
BASIL FÜRER ${ }^{a}$, ANDREAS LOCHBIHLER $^{b}$, JOSHUA SCHNEIDER $^{c}$, AND DMITRIY TRAYTEL $^{d}$ \\ ${ }^{a}$ Department of Computer Science, ETH Zürich, Switzerland \\ ${ }^{b}$ Digital Asset (Switzerland) GmbH, Zurich, Switzerland \\ e-mail address: mail@andreas-lochbihler.de \\ ${ }^{c}$ Institute of Information Security, Department of Computer Science, ETH Zürich, Switzerland \\ e-mail address: joshua.schneider@inf.ethz.ch \\ ${ }^{d}$ Department of Computer Science, University of Copenhagen, Denmark \\ e-mail address: traytel@di.ku.dk
}

\begin{abstract}
The functorial structure of type constructors is the foundation for many definition and proof principles in higher-order logic (HOL). For example, inductive and coinductive datatypes can be built modularly from bounded natural functors (BNFs), a class of well-behaved type constructors. Composition, fixpoints, and-under certain conditions - subtypes are known to preserve the BNF structure. In this article, we tackle the preservation question for quotients, the last important principle for introducing new types in HOL. We identify sufficient conditions under which a quotient inherits the BNF structure from its underlying type. Surprisingly, lifting the structure in the obvious manner fails for some quotients, a problem that also affects the quotients of polynomial functors used in the Lean proof assistant. We provide a strictly more general lifting scheme that supports such problematic quotients. We extend the Isabelle/HOL proof assistant with a command that automates the registration of a quotient type as a BNF, reducing the proof burden on the user from the full set of BNF axioms to our inheritance conditions. We demonstrate the command's usefulness through several case studies.
\end{abstract}

\section{INTRODUCTION}

The functorial structure of type constructors forms the basis for many definition and proof principles in proof assistants. Examples include datatype and codatatype definitions [ACH19, $\mathrm{BHL}^{+} 14$, TPB12], program synthesis [CDM13, HK13, LL19], generalized term rewriting [Soz10], and reasoning based on representation independence [BLS20, HK13, KP19] and about effects [Loc19, LS16].

A type constructor becomes a functor through a mapper operation that lifts functions on the type arguments to the constructed type. The mapper must be functorial, i.e., preserve identity functions (id) and distribute over function composition (o). For example, the list

Key words and phrases: Inductive and coinductive datatypes, quotient types, functors, higher-order logic, proof assistants. 
type constructor list $^{1}$ has the well-known mapper map :: $(\alpha \rightarrow \beta) \rightarrow \alpha$ list $\rightarrow \beta$ list, which applies the given function to every element in the given list. It is functorial:

$$
\text { map id }=\text { id } \quad \operatorname{map} g \circ \operatorname{map} f=\operatorname{map}(g \circ f)
$$

Most applications of functors can benefit from even richer structures. In this article, we focus on bounded natural functors (BNFs) [TPB12]. A BNF comes with additional setter operators that return sets of occurring elements, called atoms, for each type argument (Section 2.1). The setters must be natural transformations, i.e., commute with the mapper, and bounded, i.e., have a fixed cardinality bound on the sets they return. For example, lists are a BNF with the setter set : $\alpha$ list $\rightarrow \alpha$ set, which returns the set of elements in a list. It satisfies set $\circ$ map $f=f\left\langle_{-}\right\rangle \circ$ set, where $f\left\langle_{-}\right\rangle$denotes the function that maps a set $X$ to $f\langle X\rangle=\{f x \mid x \in X\}$, i.e., the image of $X$ under $f$. Moreover, set $x s$ is always a finite set because lists are finite sequences.

Originally, BNFs were introduced for modularly constructing datatypes and codatatypes $\left[\mathrm{BHL}^{+} 14\right]$ in the Isabelle/HOL proof assistant. Although (co)datatypes are still the most important use case, the BNF structure is used nowadays in other contexts such as reasoning via free theorems [LSBM19] and transferring theorems between types [Kun16,LS18].

Several type definition principles in HOL preserve the BNF structure: composition (e.g., ( $\alpha$ list) list), datatypes and codatatypes [TPB12], and-under certain conditionssubtypes [Bie15,LS18]. Subtypes include records and type copies. Accordingly, when a new type constructor is defined via one of these principles from an existing BNF, then the new type automatically comes with a mapper and setters and with theorems for the BNF properties.

One important type definition principle is missing above: quotients [Hom05, HK13, KU11, Pau06, Slo97]. A quotient type (Section 2.2) identifies elements of an underlying type according to a (partial) equivalence relation $\sim$. That is, the quotient type is isomorphic to the equivalence classes of $\sim$. For example, unordered pairs $\alpha$ upair are the quotient of ordered pairs $\alpha \times \alpha$ and the equivalence relation $\sim_{\text {upair }}$ generated by $(x, y) \sim_{\text {upair }}(y, x)$. Similarly, finite sets, bags, and cyclic lists are quotients of lists where the equivalence relation permutes or duplicates the list elements as needed.

In this article, we answer the question when and how a quotient type inherits its underlying type's BNF structure. It is well known that a quotient preserves the functorial properties if the underlying type's mapper preserves $\sim$; then the quotient type's mapper is simply the lifting of the underlying type's mapper to equivalence classes [ACH19].

For setters, the situation is more complicated. Adámek et al. [AGT10] call a functor sound if it preserves empty intersections. All BNFs are sound. However, we discovered that if the setters are defined as one would expect for a quotient, then the resulting functor may be unsound. To repair the situation, we characterize the setters in terms of the mapper and identify a definition scheme for the setters that results in sound functors. We then derive sufficient conditions on the equivalence relation $\sim$ for the BNF properties to be preserved for these definitions (Section 3).

Moreover, we have implemented an Isabelle/HOL command that automates the registration of a quotient type as a BNF (Section 4); the user merely needs to discharge the conditions on $\sim$. One of the conditions, subdistributivity, often requires considerable proof effort, though. We therefore developed a novel sufficient criterion using confluent relations that simplifies the proofs in our case studies (Section 3.4). Our implementation is distributed

\footnotetext{
${ }^{1}$ Type constructors are written postfix in this article.
} 
with the Isabelle2021 release. Some of the examples in this article are only available in Isabelle's development repository ${ }^{2}$ and will be part of the forthcoming Isabelle release.

Contributions. The main contributions of this article are the following:

(1) We identify sufficient criteria for when a quotient type preserves the BNF properties of the underlying type. Registering a quotient as a BNFs allows (co)datatypes to nest recursion through it. Consider for example node-labeled unordered binary trees

datatype $u b t r e e=$ Leaf $\mid$ Node nat (ubtree upair)

BNF use cases beyond datatypes benefit equally.

(2) In particular, we show that the straightforward definitions would cause the functor to be unsound, and find better definitions that avoid unsoundness. This problem is not limited to BNFs. The lifting operations for Lean's quotients of polynomial functors [ACH19] also suffer from unsoundness and our repair applies to them as well (Section 5.2). We show in Section 5.2 that unsoundness hinders modular proofs.

(3) We propose a sufficient criterion on $\sim$ for subdistributivity, which is typically the most difficult BNF property to show. We show with several examples that the criterion is applicable in practice and yields relatively simple proofs.

(4) We have implemented an Isabelle/HOL command to register the quotient as a BNF once the user has discharged the conditions on $\sim$. The command also generates proof rules for transferring theorems about the BNF operations from the underlying type to the quotient (Section 4.2). Several case studies demonstrate the command's usefulness. Some examples reformulate well-known BNFs as quotients (e.g., unordered pairs, distinct lists, finite sets). Others formally prove the BNF properties for the first time, e.g., cyclic lists, the free idempotent monoid, and regular expressions modulo ACI. These examples become part of the collection of formalized BNFs and can thus be used in datatype definitions and other BNF applications.

Example 1.1. To illustrate our contributions' usefulness, we consider linear dynamic logic (LDL) [DV13], an extension of linear temporal logic with regular expressions. LDL's syntax is usually given as two mutually recursive datatypes of formulas and regular expressions [DV13, BKT17]. Here, we opt for nested recursion, which has the modularity benefit of being able to formalize regular expressions separately. We define regular expressions $\alpha$ re:

$$
\begin{aligned}
\text { datatype } \alpha \text { re } & =\text { Zero } \mid \text { Eps } \mid \text { Atom } \alpha \\
& \mid \text { Alt }(\alpha r e)(\alpha r e) \mid \text { Conc }(\alpha r e)(\alpha r e) \mid \operatorname{Star}(\alpha r e)
\end{aligned}
$$

Often, it is useful to consider regular expressions modulo some syntactic equivalences. For example, identifying expressions modulo the associativity, commutativity, and idempotence (ACI) of the alternation constructor Alt results in a straightforward construction of deterministic finite automata from regular expressions via Brzozowski derivatives [NT14]. We define the ACI-equivalence $\sim_{a c i}$ as the least congruence relation satisfying:

$$
\text { Alt }(\text { Alt } r s) t \sim_{a c i} \text { Alt } r(\text { Alt } s t) \quad \text { Alt } r s \sim_{a c i} \text { Alt } s r \quad \text { Alt } r r \sim_{a c i} r
$$

Next, we define the quotient type of regular expressions modulo ACI $\alpha r_{a c i}$ and the datatype of LDL formulas $l d l$, which uses nested recursion through $\alpha r e_{a c i}$.

\footnotetext{
${ }^{2}$ https://isabelle.in.tum.de/repos/isabelle, revision be11fe268b33
} 


$$
\begin{aligned}
& \text { quotient_type } \alpha r e_{a c i}=\alpha r e / \sim_{a c i} \\
& \text { datatype } l d l=\text { Prop string }|\operatorname{Neg} l d l| \text { Conj } l d l l d l \mid \text { Match }\left(l d l r e_{a c i}\right)
\end{aligned}
$$

For the last declaration to succeed, Isabelle must know that $\alpha r e_{a c i}$ is a BNF. We will show in Section 3.4 how our work allows us to lift $\alpha$ re's BNF structure to $\alpha$ re $e_{a c i}$.

This article extends and revises the homonymous IJCAR conference paper [FLST20]. Specifically, the article newly describes the interaction of quotients and non-emptiness witnesses [BPT15], an additional piece of information tracked as part of the BNF structure and used to prove non-emptiness of inductive datatypes, which is a requirement for introducing new types in HOL (Section 3.5). We also show how to lift the BNF structure to partial quotients by combining our constructions for quotients with the ones for subtypes, and discuss limitations of this approach (Section 3.6). Moreover, we include several previously omitted pen-and-paper proofs of our results, whose formalized counterparts are implemented as tactics as part of our Isabelle command to automate the lifting. We also give a more detailed description of our command's interface (Section 4.1) and formalize several new examples, some of which required us to extend our results' scope. Notably, the new Example 3.26 uses the new Lemma 3.11 and the updated Theorem 3.21, which generalizes the corresponding Theorem 4 from the conference paper.

\section{BACKGROUND}

We work in Isabelle/HOL, Isabelle's variant of classical higher-order logic - a simply typed theory with Hilbert choice and rank-1 polymorphism. We refer to a textbook for a detailed introduction to Isabelle/HOL [NK14] and only summarize relevant notation here.

Types are built from type variables $\alpha, \beta, \ldots$ via type constructors. A type constructor can be nullary (nat) or have some type arguments ( $\alpha$ list, $\alpha$ set, $(\alpha, \beta)$ upair). Type constructor application is written postfix. Exceptions are the binary type constructors for sums $(+)$, products $(\times)$, and functions $(\rightarrow)$, all written infix. Terms are built from variables

$x, y, \ldots$ and constants $\mathrm{c}, \mathrm{d}, \ldots$ via lambda-abstractions $\lambda x . t$ and applications $t u$. The sum type's embeddings are Inl and Inr and the product type's projections are fst and snd.

The primitive way of introducing new types in HOL is to take a non-empty subset of an existing type. For example, the type of lists could be defined as the set of pairs ( $n::$ nat, $f::$ nat $\rightarrow \alpha$ ) where $n$ is the list's length and $f i$ is the list's $i$ th element for $i<n$ and some fixed unspecified element of type $\alpha$ for $i \geq n$. To spare the users from such low-level encodings, Isabelle/HOL offers higher-level mechanisms for introducing new types, which are internally reduced to primitive subtyping. In fact, lists are defined as an inductive datatype $\alpha$ list $=[] \mid \alpha \# \alpha$ list, where [] is the empty list and \# is the infix list constructor. Recursion in datatypes and their coinductive counterparts may take place only under well-behaved type constructors, the bounded natural functors (Section 2.1). Quotient types (Section 2.2) are another high-level mechanism for introducing new types.

For $n$-ary definitions, we use the vector notation $\bar{x}$ to denote the sequence $x_{1}, \ldots, x_{n}$, where $n$ is clear from the context. Vectors spanning several variables indicate repetition with synchronized indices. For example, $\operatorname{map}_{F} \overline{(g \circ f)}$ abbreviates $\operatorname{map}_{F}\left(g_{1} \circ f_{1}\right) \ldots\left(g_{n} \circ f_{n}\right)$. Abusing notation slightly, we write $\bar{\alpha} \rightarrow \beta$ for the $n$-ary function type $\alpha_{1} \rightarrow \cdots \rightarrow \alpha_{n} \rightarrow \beta$.

To simplify notation, we identify the type of binary predicates $\alpha \rightarrow \beta \rightarrow$ bool and sets of pairs $(\alpha \times \beta)$ set, and write $\alpha \otimes \beta$ for both. These types are different in Isabelle/HOL and 
the BNF ecosystem works with binary predicates. The identification allows us to mix set and relation operations, e.g., the subset relation $\subseteq$ and relation composition $\bullet$ (both written infix).

2.1. Bounded Natural Functors. A bounded natural functor (BNF) [TPB12] is an $n$ ary type constructor $\bar{\alpha} F$ equipped with the following polymorphic constants. Here and elsewhere, $i$ implicitly ranges over $\{1, \ldots, n\}$ :

$$
\begin{array}{ll}
\operatorname{map}_{F}:: \overline{(\alpha \rightarrow \beta)} \rightarrow \bar{\alpha} F \rightarrow \bar{\beta} F & \operatorname{bd}_{F}:: \operatorname{cardinal}_{F} \\
\operatorname{set}_{F, i}:: \bar{\alpha} F \rightarrow \alpha_{i} \text { set for all } i & \operatorname{rel}_{F}:: \overline{(\alpha \otimes \beta)} \rightarrow \bar{\alpha} F \otimes \bar{\beta} F
\end{array}
$$

The shape and content intuition [TPB12] is a useful way of thinking about elements of $\bar{\alpha} F$. The mapper $\operatorname{map}_{F}$ leaves the shape unchanged but modifies the contents by applying

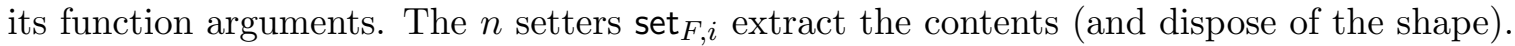
For example, the shape of a list is given by its length, which map preserves. The cardinal bound $b_{F}$ is a fixed bound on the number of elements returned by set $F, i$. Cardinal numbers are represented in HOL using particular well-ordered relations [BPT14] over a large-enough type (specific to $F$ ). We mention the bounds and cardinal numbers only for completeness; they are not interesting for this article. Finally, the relator rel $_{F}$ lifts relations on the type arguments to a relation on $\bar{\alpha} F$ and $\bar{\beta} F$. Thereby, it only relates elements of $\bar{\alpha} F$ and $\bar{\beta} F$ that have the same shape.

The BNF constants must satisfy the following properties:

$$
\begin{aligned}
\text { MAP_ID } & \operatorname{map}_{F} \overline{\mathrm{id}}=\mathrm{id} \\
\text { MAP_COMP } & \operatorname{map}_{F} \bar{g} \circ \operatorname{map}_{F} \bar{f}=\operatorname{map}_{F} \overline{(g \circ f)} \\
\text { SET_MAP } & \operatorname{set}_{F, i} \circ \operatorname{map}_{F} \bar{f}=f_{i}\left\langle\left\langle_{-} \circ \operatorname{set}_{F, i}\right.\right. \\
\text { MAP_CONG } & \left(\forall i . \forall z \in \operatorname{set}_{F, i} x . f_{i} z=g_{i} z\right) \Longrightarrow \operatorname{map}_{F} \bar{f} x=\operatorname{map}_{F} \bar{g} x \\
\text { SET_BD } & \left|\operatorname{set}_{F, i} x\right| \leq_{o} \mathrm{bd}_{F} \\
\text { BD } & \text { infinite_card bd }_{F} \\
\text { IN_REL } & \text { rel } F \bar{R} x y=\exists z .\left(\forall i . \operatorname{set}_{F, i} z \subseteq R_{i}\right) \wedge \operatorname{map} \overline{\text { fst }} z=x \wedge \operatorname{map} \overline{\text { snd }} z=y \\
\text { REL_COMP } & \text { rel } F \bar{R} \bullet \operatorname{rel}_{F} \bar{S} \subseteq \operatorname{rel}_{F} \overline{(R \bullet S)}
\end{aligned}
$$

Properties MAP_ID and MAP_COMP capture the mapper's functoriality; SET_MAP the setters' naturality. Moreover, the mapper and the setters must agree on what they identify as content (MAP_CONG). Any set returned by $\operatorname{set}_{F, i}$ must be bounded (SET_BD); the operator $\leq_{o}$ compares cardinal numbers [BPT14]. The bound is required to be infinite (BD), which simplifies arithmetics. The relator can be expressed in terms of the mapper and the setter (IN_REL) and must distribute over relation composition (REL_COMP). The other inclusion, namely $\operatorname{rel}_{F} \overline{(R \bullet S)} \subseteq \operatorname{rel}_{F} \bar{R} \bullet \operatorname{rel}_{F} \bar{S}$, follows from these properties. We refer to REL_COMP as subdistributivity because it only requires one inclusion. In principle, the setter can also be expressed in terms of the mapper as the least set satisfying the congruence rule MAP_CONG. We rely on this fact in Section 3. Making the setters and the relator part of the BNF structure (rather than defining everything from the mapper) simplifies the statement of the BNF properties.

A useful derived operator is the action on sets $F:: \overline{\alpha s e t} \rightarrow \bar{\alpha} F$ set, which generalizes the type constructor's action on its type arguments. Formally, $F \bar{A}=\left\{x \mid \forall i\right.$. $\left.\operatorname{set}_{F, i} x \subseteq A_{i}\right\}$. Note that we can write $z \in\left[F \bar{R}\right.$ to replace the equivalent $\forall i$. set $F, i=R_{i}$ in IN_REL.

Most basic types are BNFs, notably, sum and product types. BNFs are closed under composition, e.g., $1+\alpha \times \beta$ is a BNF with the mapper $\lambda f g$. $\operatorname{map}_{1+}\left(\operatorname{map}_{\times} f g\right)$, where 1 is the unit type (consisting of the single element $\star$ ) and $\operatorname{map}_{1+} h=\operatorname{map}_{+}$id $h$. Moreover, 
BNFs support fixpoint operations, which correspond to (co)datatypes, and are closed under them [TPB12]. For instance, the datatype command internally computes a least solution for the fixpoint type equation $\beta=1+\alpha \times \beta$ to define the $\alpha$ list type. Closure means that the resulting datatype, here $\alpha$ list, is equipped with the BNF structure, specifically the mapper map. Also subtypes inherit the BNF structure under certain conditions (Section 3.6). For example, the subtype $\alpha$ nelist of non-empty lists $\{x s:: \alpha$ list $\mid x s \neq[]\}$ is a BNF.

2.2. Quotient types. An equivalence relation $\sim$ on a type $T$ partitions the type into equivalence classes. Isabelle/HOL supports the definition of the quotient type $Q=T / \sim$, which yields a new type $Q$ isomorphic to the set of equivalence classes [KU11]. For example, consider $\sim_{f s e t}$ that relates two lists if they have the same set of elements, i.e., $x s \sim_{f s e t} y s$ iff set $x s=$ set ys. The following command defines the type $\alpha$ fset of finite sets as a quotient of lists:

$$
\text { quotient_type } \alpha \text { fset }=\alpha \text { list } / \sim_{f s e t}
$$

This command requires a proof that $\sim_{f s e t}$ is, in fact, an equivalence relation. The relationship between a quotient type $Q$ and the underlying type $T$ is formally captured by the correspondence relation $\operatorname{cr}_{Q}:: T \otimes Q$. For example, $(x s, X) \in \mathrm{cr}_{\text {fset }}$ iff the list $x s$ is a representative of the finite set $X$, i.e., $X$ corresponds to the unique equivalence class that contains $x s$.

The Lifting and Transfer tools [HK13, Kun16] automate the lifting of definitions and theorems from the raw type $T$ to the quotient $Q$. For example, the image operation on finite sets can be obtained by lifting the list mapper map using the command

$$
\text { lift_definition fimage }::(\alpha \rightarrow \beta) \rightarrow \alpha \text { fset } \rightarrow \beta \text { fset is map }
$$

Lifting is only possible for terms that respect the quotient. For fimage, respectfulness states that map $f x s \sim_{f s e t}$ map $f$ ys whenever $x s \sim_{f s e t} y s$.

Lifting and Transfer are based on transfer rules that relate two terms of possibly different types. The lift_definition command automatically proves the transfer rule

$$
\text { (map, fimage }) \in\left((=) \Leftrightarrow \mathrm{cr}_{\text {fset }} \Leftrightarrow \mathrm{cr}_{\text {fset }}\right)
$$

where $R \Leftrightarrow S$ (right-associative) relates two functions iff they map $R$-related arguments to $S$-related results. The meaning of the above rule is that applying map $f$ to a list representing the finite set $X$ results in a list that represents fimage $f X$, for all $f$. The transfer rule's relation $(=) \Leftrightarrow \mathrm{cr}_{\text {fset }} \Leftrightarrow \mathrm{cr}_{\text {sset }}$ is constructed according to the types of the related terms. This enables the composition of transfer rules to relate larger terms. For instance, the Transfer tool derives the following equivalence using the above and other transfer rules:

$$
(\forall x s \text {. set }(\text { map id } x s)=\text { set } x s) \longleftrightarrow(\forall X \text {. fimage id } X=X)
$$

Thus, one can prove the equation $\forall X$. fimage id $X=X$ by reasoning about lists.

Proper equivalence relations are reflexive. Therefore, every element of the type $T$ is part of exactly one equivalence class. It is also possible to define a partial quotient from a partial equivalence relation, which might not be reflexive. The quotient_type command and the Lifting and Transfer tools support partial quotients. Note that partial quotients subsume subtypes (take the restriction of equality to the subset as the partial equivalence). 


\section{Quotients of Bounded Natural Functors}

We develop the theory for when a quotient type inherits the underlying type's BNF structure. We consider the quotient $\bar{\alpha} Q=\bar{\alpha} F / \sim$ of an $n$-ary BNF $\bar{\alpha} F$ over an equivalence relation $\sim$ on $\bar{\alpha} F$. The first idea is to define $\operatorname{map}_{Q}$ and $\operatorname{set}_{Q, i}$ in terms of $F$ 's operations:

$$
\begin{aligned}
& \text { quotient_type } \bar{\alpha} Q=\bar{\alpha} F / \sim \\
& \text { lift_definition } \operatorname{map}_{Q}:: \overline{(\alpha \rightarrow \beta)} \rightarrow \bar{\alpha} Q \rightarrow \bar{\beta} Q \text { is } \operatorname{map}_{F} \\
& \text { lift_definition } \operatorname{set}_{Q, i}:: \bar{\alpha} Q \rightarrow \alpha_{i} \text { set is } \operatorname{set}_{F, i}
\end{aligned}
$$

These three commands require the user to discharge the following proof obligations:

$$
\begin{aligned}
\text { equivp } & \sim \\
x \sim y \Longrightarrow \operatorname{map}_{F} \bar{f} x & \sim \operatorname{map}_{F} \bar{f} y \\
x \sim y \Longrightarrow \operatorname{set}_{F, i} x & =\operatorname{set}_{F, i} y
\end{aligned}
$$

The first two conditions are as expected: $\sim$ must be an equivalence relation, by (3.1), and compatible with $F$ 's mapper, by (3.2), i.e., $\operatorname{map}_{F}$ preserves $\sim$. The third condition, however, demands that equivalent values contain the same atoms. This rules out many practical examples including the following simplified (and therefore slightly artificial) one.

Example 3.1. Consider $\alpha F_{P}=\alpha+\alpha$ with the equivalence relation $\sim_{P}$ generated by $\operatorname{Inl} x \sim_{P} \operatorname{Inl} y$, where $\operatorname{lnl}$ is the sum type's left embedding. That is, $\sim_{P}$ identifies all values of the form $\operatorname{lnl} z$ and thus $\alpha Q_{P}=\alpha F_{P} / \sim_{P}$ is isomorphic to the type $1+\alpha$. However, $\ln \mid x$ and $\operatorname{Inl} y$ have different sets of atoms $\{x\}$ and $\{y\}$, assuming $x \neq y$.

We now derive better definitions for the setters and conditions under which they preserve the BNF properties. To that end, we characterize setters in terms of the mapper (Section 3.1). Using this characterization, we derive the relationship between $\operatorname{set}_{Q, i}$ and $\operatorname{set}_{F, i}$ and identify the conditions on $\sim$ (Section 3.2). Next, we do the same for the relator (Section 3.3). We thus obtain the conditions under which $\bar{\alpha} Q$ preserves $F$ 's BNF properties.

One of the conditions, the relator's subdistributivity over relation composition, is often difficult to show directly in practice. We therefore present an easier-to-establish criterion for the special case where a confluent rewrite relation $\rightsquigarrow$ generates $\sim$ (Section 3.4).

Finally, we discuss the interaction of quotients with non-emptiness witnesses (Section 3.5), an additional piece of information tracked by BNFs, and the generalization to partial quotients, where $\sim$ is a partial equivalence relation, i.e., not necessarily reflexive (Section 3.6).

3.1. Characterization of the BNF setter. We now characterize set ${ }_{F, i}$ in terms of map $F$ for an arbitrary BNF $\bar{\alpha} F$. Observe that $F$ 's action $F \bar{A}$ on sets contains all values that can be built with atoms from $\bar{A}$. Hence, $\operatorname{set}_{F, i} x$ is the smallest set $A_{i}$ such that $x$ can be built from atoms in $A_{i}$. Formally, the next equation follows directly from the definition of $F$ :

$$
\operatorname{set}_{F, i} x=\bigcap\left\{A_{i} \mid x \in \bar{F} \overline{\mathrm{UNIV}} A_{i} \overline{\mathrm{UNIV}}\right\}
$$

Only atoms of type $\alpha_{i}$ are restricted; all atoms of other types $\alpha_{j}$ may come from UNIV, the set of all elements of type $\alpha_{j}$. Moreover, $F$ can be defined without set ${ }_{F, i}$, namely by trying to distinguish values using the mapper. Informally, $x$ contains atoms not from $\bar{A}$ iff $\operatorname{map}_{F} \bar{f} x$ differs from $\operatorname{map}_{F} \bar{g} x$ for some functions $\bar{f}$ and $\bar{g}$ that agree on $\bar{A}$. Hence, we obtain

$$
F \bar{A}=\left\{x \mid \forall \bar{f} \bar{g} .\left(\forall i . \forall a \in A_{i} . f_{i} a=g_{i} a\right) \longrightarrow \operatorname{map}_{F} \bar{f} x=\operatorname{map}_{F} \bar{g} x\right\},
$$


where $f_{i}, g_{i}:: \alpha_{i} \rightarrow 1+\alpha_{i}$. The range type $1+\alpha_{i}$ adds a new atom $\circledast=\ln \mid \star$ to the atoms of type $\alpha_{i}$. Thus $1+\alpha_{i}$ contains at least two atoms, as all HOL types are inhabited, and $f_{i}$ and $g_{i}$ can therefore meaningfully distinguish atoms (for singleton types $\bar{\alpha}$, the right hand side would hold trivially if $f_{i}, g_{i}$ had type the $\alpha_{i} \rightarrow \alpha_{i}$ because there is only one such function). We write $\mathfrak{e}:: \alpha \rightarrow 1+\alpha$ for the embedding of $\alpha$ into $1+\alpha$ (i.e., $\mathfrak{e}=\ln r$ ).

Proof. From left to right is trivial with MAP_CONG. So let $x$ be such that $\operatorname{map}_{F} \bar{f} x=$ $\operatorname{map}_{F} \bar{g} x$ whenever $f_{i} a=g_{i} a$ for all $a \in A_{i}$ and all $i$. By the definition of $F$, it suffices to show that $\operatorname{set}_{F, i} x \subseteq A_{i}$. Set $f_{i} a=\mathfrak{e} a$ if $a \in A_{i}$ and $f_{i} a=\circledast$ for $a \in A_{i}$, and $g_{i}=\mathfrak{e}$. Then,

$$
\begin{aligned}
f_{i}\left\langle\operatorname{set}_{F, i} x\right\rangle & =\operatorname{set}_{F, i}\left(\operatorname{map}_{F} \bar{f} x\right) & & \text { by SET_MAP } \\
& =\operatorname{set}_{F, i}\left(\operatorname{map}_{F} \bar{g} x\right) & & \text { by choice of } x \text { as } \bar{f} \text { and } \bar{g} \text { agree on } \bar{A} \\
& =\mathfrak{e}\left\langle\operatorname{set}_{F, i} x\right\rangle & & \text { by SET_MAP }
\end{aligned}
$$

Therefore, $\forall a \in \operatorname{set}_{F, i} x . \exists y . f_{i} a=\mathfrak{e} y$, i.e., $\operatorname{set}_{F, i} x \subseteq A_{i}$ by $f_{i}$ 's definition.

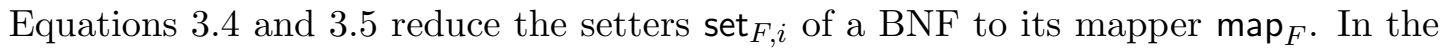
next section, we will use this characterization to derive a definition of $\operatorname{set}_{Q, i}$ in terms of $\operatorname{set}_{F, i}$. However, this definition does not give us naturality out of the box.

Example 3.2 [AGT10, Example 4.2, part iii]. Consider the functor $\alpha F_{\text {seq }}=n a t \rightarrow \alpha$ of infinite sequences with $x \sim_{a e} y$ whenever $\{n \mid x n \neq y n\}$ is finite. That is, two sequences are equivalent iff they are equal almost everywhere. Conditions (3.1) and (3.2) hold, but not the naturality for the corresponding $\operatorname{map}_{Q}$ and $\operatorname{set}_{Q}$.

Gumm [Gum05] showed that $\operatorname{set}_{F}$ as defined in terms of (3.4) and (3.5) is a natural transformation iff $F$ preserves wide intersections and preimages, i.e.,

$$
\begin{gathered}
F \overline{(\bigcap \mathcal{A})}=\bigcap\left\{\bar{F} \bar{A} \mid \forall i . A_{i} \in \mathcal{A}_{i}\right\} \\
F \overline{\left(f^{-1}\langle A\rangle\right)}=\left(\operatorname{map}_{F} \bar{f}\right)^{-1}\langle\bar{F} \bar{A}\rangle
\end{gathered}
$$

where $f^{-1}\langle A\rangle=\{x \mid f x \in A\}$ denotes the preimage of $A$ under $f$. Then, $F \bar{A}=\{x \mid$ $\forall i$. $\left.\operatorname{set}_{F, i} x \subseteq A_{i}\right\}$ holds. The quotient in Example 3.2 does not preserve wide intersections.

In theory, we have now everything we need to define the BNF operations on the quotient $\bar{\alpha} Q=\bar{\alpha} F / \sim$ : Define $\operatorname{map}_{Q}$ as the lifting of $\operatorname{map}_{F}$. Define $Q$ and $\operatorname{set}_{Q, i}$ using (3.5) and (3.4) in terms of $\operatorname{map}_{Q}$, and the relator via IN_REL. Prove that $\bar{Q}$ preserves preimages and wide intersections. Prove that rel $_{Q}$ satisfies subdistributivity (REL_COMP).

Unfortunately, the definitions and the preservation conditions are phrased in terms of $Q$, not in terms of $F$ and $\sim$. It is therefore unclear how $\operatorname{set}_{Q, i}$ and $\operatorname{rel}_{Q}$ relate to $\operatorname{set}_{F, i}$ and $\mathrm{rel}_{F}$. In practice, understanding this relationship is important: we want to express the BNF operations and discharge the proof obligations in terms of $F$ 's operations and later use the connection to transfer properties from set $F$ and $\operatorname{rel}_{F}$ to $\operatorname{set}_{Q}$ and $\operatorname{rel}_{Q}$. We will work out the precise relationships for the setters in Section 3.2 and for the relator in Section 3.3.

3.2. The quotient's setter. We relate $Q$ 's setters to $F$ 's operations and $\sim$. We first look at $Q$, which characterizes $\operatorname{set}_{Q, i}$ via (3.4). Let $[x]_{\sim}=\{y \mid x \sim y\}$ denote the equivalence class that $x:: \bar{\alpha} F$ belongs to, and $[A]_{\sim}=\left\{[x]_{\sim} \mid x \in A\right\}$ denote the equivalence classes 
of elements in $A$. We identify the values of $\bar{\alpha} Q$ with $\bar{\alpha} F$ 's equivalence classes. Then, it

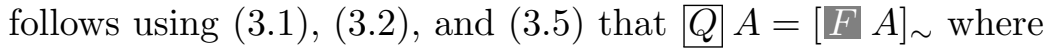

$$
F \bar{A}=\left\{x \mid \forall \bar{f} \bar{g} .\left(\forall i . \forall a \in A_{i} . f_{i} a=g_{i} a\right) \longrightarrow \operatorname{map}_{F} \bar{f} x \sim \operatorname{map}_{F} \bar{g} x\right\}
$$

with $f_{i}, g_{i}:: \alpha_{i} \rightarrow 1+\alpha_{i}$. Equation 3.8 differs from (3.5) only in that the equality in $\operatorname{map}_{F} \bar{f} x=\operatorname{map}_{F} \bar{g} x$ is replaced by $\sim$. Clearly $\left[[F \bar{A}]_{\sim} \subseteq[E \bar{A}]_{\sim}\right.$. The converse holds for non-empty sets $A_{i}$, as shown next.

Lemma 3.3. If $A_{i} \neq\{\}$ for all $i$, then $[F \bar{A}]_{\sim} \subseteq[E \bar{A}]_{\sim}$.

Proof. Since $A_{i}$ is non-empty, fix $a_{i} \in A_{i}$ for all $i$. Let $x \in F \bar{A}$ and consider $y=\operatorname{map}_{F} \bar{h} x$ where $h_{i} a=a$ if $a \in A_{i}$ and $h_{i} a=a_{i}$ otherwise. Let $\mathfrak{e}^{-1}$ denote the left-inverse of $\mathfrak{e}$. Then,

$$
\begin{aligned}
& x=\operatorname{map}_{F} \overline{\left(\mathfrak{e}^{-1} \circ \mathfrak{e}\right)} x \quad \text { by MAP_ID and } \mathfrak{e}^{-1} \circ \mathfrak{e}=\mathrm{id} \\
& =\operatorname{map}_{F} \overline{\mathfrak{e}^{-1}}\left(\operatorname{map}_{F} \overline{\mathfrak{e}} x\right) \quad \text { by MAP_COMP } \\
& \sim \operatorname{map}_{F} \overline{\mathfrak{e}^{-1}}\left(\operatorname{map}_{F} \overline{(\mathfrak{e} \circ h)} x\right) \quad \text { by (3.8) and (3.2) as } \mathfrak{e} a=\left(\mathfrak{e} \circ h_{i}\right) a \text { for } a \in A_{i} \\
& =\operatorname{map}_{F} \overline{\left(\mathfrak{e}^{-1} \circ \mathfrak{e} \circ h\right)} x=y \quad \text { by MAP_COMP and } \mathfrak{e}^{-1} \circ \mathfrak{e}=\mathrm{id}
\end{aligned}
$$

It therefore suffices to show that $y \in F \bar{A}$. Let $\bar{f}$ and $\bar{g}$ with $f_{i}, g_{i}:: \alpha_{i} \rightarrow 1+\alpha_{i}$ such that $f_{i} a=g_{i} a$ for all $a \in A_{i}$. Then $f_{i} \circ h_{i}=g_{i} \circ h_{i}$ as the range of $h_{i}$ is $A_{i}$. So $\operatorname{map}_{F} \bar{f} y=\operatorname{map}_{F} \overline{(f \circ h)} x=\operatorname{map}_{F} \overline{(g \circ h)} x=\operatorname{map}_{F} \bar{g} y$. Thus $y \in F \bar{A}$.

However, this inclusion $[F \bar{A}]_{\sim} \subseteq[F \bar{A}]_{\sim}$ may fail for empty sets $A_{i}$, as the next example shows.

Example 3.4 (Example 3.1 continued). For the example viewing $1+\alpha$ as a quotient of $\alpha F_{P}=\alpha+\alpha$ via $\sim_{P}$, we have $[\operatorname{lnl} x]_{\sim_{P}} \in\left[F_{P}\{\}\right]_{\sim_{P}}$ because $\operatorname{map}_{F_{P}} f(\operatorname{lnl} x)=$ $\operatorname{lnl}(f x) \sim_{P} \operatorname{lnl}(g x)=\operatorname{map}_{F_{P}} g(\operatorname{Inl} x)$ for all $f$ and $g$. Yet $F_{P}\{\}$ is empty, and so is $\left[F_{P}\{\}\right]_{\sim_{P}}$.

To avoid the problematic case of empty sets, we change types: instead of $\bar{\alpha} F / \sim$, we consider the quotient $\overline{(1+\alpha)} F / \sim$. Then, we have the following equivalence:

Lemma 3.5. $F \bar{A}=\left\{x \mid\left[\operatorname{map}_{F} \overline{\mathfrak{e}} x\right]_{\sim} \in[\overline{F(\{\circledast\} \cup \mathfrak{e}\langle A\rangle)}]_{\sim}\right\}$.

Proof. For the left to right direction, let $x \in F \bar{A}$ and set $f_{i} y=\mathfrak{e} y$ for $y \in A_{i}$ and $f_{i} y=\circledast$ for $y \notin A_{i}$. Then, $\operatorname{set}_{F, i}\left(\operatorname{map}_{F} \bar{f} x\right)=f_{i}\left\langle\operatorname{set}_{F, i} x\right\rangle$ by the naturality of $\operatorname{set}_{F, i}$ and $f_{i}\langle B\rangle \subseteq\{\circledast\} \cup \mathfrak{e}\left\langle A_{i}\right\rangle$ by $f_{i}$ 's definition for any $B$. Hence map $\bar{f} x \in \overline{F(} \overline{(\{\circledast\} \cup \mathfrak{e}\langle A\rangle)}$ as $F \bar{C}=\left\{x \mid \forall i\right.$. $\left.\operatorname{set}_{F, i} x \subseteq C_{i}\right\}$ by definition. So, $\left[\operatorname{map}_{F} \overline{\mathfrak{e}} x\right]_{\sim} \in[\overline{F(\{\otimes\} \cup \mathfrak{e}\langle A\rangle)}]_{\sim}$ because $\operatorname{map}_{F} \overline{\mathfrak{e}} x \sim \operatorname{map} \bar{f} x$ by (3.8) and $x \in F \bar{A}$.

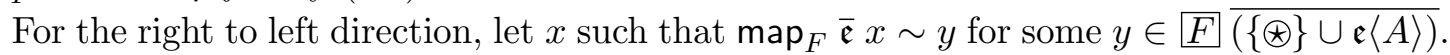
Let $\bar{f}$ and $\bar{g}$ such that $f_{i} a=g_{i} a$ for all $a \in A_{i}$ and all $i$. Then, $\operatorname{map}_{F} \bar{f} x \sim \operatorname{map}_{F} \bar{g} x$ holds by the following reasoning, where $\operatorname{map}_{1+} h$ satisfies $\operatorname{map}_{1+} h(\mathfrak{e} a)=\mathfrak{e}\left(\begin{array}{ll}h & a\end{array}\right)$ and $\operatorname{map}_{1+} h \circledast=\circledast:$

$$
\begin{aligned}
\operatorname{map}_{F} \bar{f} x & =\operatorname{map}_{F} \overline{\mathfrak{e}^{-1}}\left(\operatorname{map}_{F} \overline{\left(\operatorname{map}_{1+f}\right)}\left(\operatorname{map}_{F} \overline{\mathfrak{e}} x\right)\right) & & \text { as } f_{i}=\mathfrak{e}^{-1} \circ \operatorname{map}_{1+} f_{i} \circ \mathfrak{e} \\
& \sim \operatorname{map}_{F} \overline{\mathfrak{e}^{-1}}\left(\operatorname{map}_{F} \overline{\left(\operatorname{map}_{1+f}\right)} y\right) & & \text { by } \operatorname{map}_{F} \overline{\mathfrak{e}} x \sim y \text { and }(3.2) \\
& =\operatorname{map}_{F} \overline{\mathfrak{e}^{-1}}\left(\operatorname{map}_{F} \overline{\left(\operatorname{map}_{1+} g\right)} y\right) & & \text { by choice of } y \text { and }(3.5) \\
& \sim \operatorname{map}_{F} \overline{\mathfrak{e}^{-1}}\left(\operatorname{map}_{F} \overline{\left(\operatorname{map}_{1+} g\right)}\left(\operatorname{map}_{F} \overline{\mathfrak{e}} x\right)\right) & & \text { by } y \sim \operatorname{map}_{F} \overline{\mathfrak{e}} x \text { and }(3.2) \\
& =\operatorname{map}_{F} \bar{g} x & & \text { as } \mathfrak{e}^{-1} \circ \operatorname{map}_{1+} g_{i} \circ \mathfrak{e}=g_{i}
\end{aligned}
$$


Lemma 3.5 allows us to characterize the quotient's setters $\operatorname{set}_{Q}$ in terms of $\operatorname{set}_{F}$.

Theorem 3.6 (Setter characterization). $\operatorname{set}_{Q, i}[x]_{\sim}=\bigcap_{y \in\left[\operatorname{map}_{F} \overline{\mathfrak{e}} x\right]_{\sim}}\left\{a \mid \mathfrak{e} a \in \operatorname{set}_{F, i} y\right\}$

Proof. Recall that we defined $\operatorname{set}_{Q, i}$ by (3.4). Then

$$
\begin{array}{rlrl}
\operatorname{set}_{Q, i}[x]_{\sim} & =\bigcap\left\{A_{i} \mid[x]_{\sim} \in Q \overline{\mathrm{UNIV}} A_{i} \overline{\mathrm{UNIV}}\right\} & & \text { by }(3.4) \\
& =\bigcap\left\{A_{i} \mid[x]_{\sim} \in\left[\overline{\mathrm{UNIV}} A_{i} \overline{\mathrm{UNIV}}\right]_{\sim}\right\} & & \text { by } \bar{Q} \bar{A}=[\bar{F} \bar{A}]_{\sim} \\
& =\bigcap\left\{A_{i} \mid\left[\operatorname{map}_{F} \overline{\mathfrak{e}} x\right]_{\sim} \in\left[\overline{\mathrm{UNIV}}\left(\{\circledast\} \cup \mathfrak{e}\left\langle A_{i}\right\rangle\right) \overline{\mathrm{UNIV}}\right]_{\sim}\right\} & & \text { by Lemma } 3.5 \\
& =\bigcap\left\{A_{i} \mid\left[\operatorname{map}_{F} \overline{\mathfrak{e}} x\right]_{\sim} \in\left[\left\{y \mid \operatorname{set}_{F, i} y \subseteq\{\circledast\} \cup \mathfrak{e}\left\langle A_{i}\right\rangle\right\}\right]_{\sim}\right\} & & \text { by Definition of }[F \\
& \left.=\bigcap\left\{a \mid \mathfrak{e} a \in \operatorname{set}_{F, i} y\right\} \mid y \sim \operatorname{map}_{F} \overline{\mathfrak{e}} x\right\} & & \\
& =\bigcap_{y \in\left[\operatorname{map}_{F} \overline{\mathfrak{e}} x\right]_{\sim}\left\{a \mid \mathfrak{e} a \in \operatorname{set}_{F, i} y\right\}} &
\end{array}
$$

Example 3.7 (Example 3.4 continued). For the example viewing $1+\alpha$ as a quotient of $\alpha F_{P}=\alpha+\alpha$ via $\sim_{P}$, Theorem 3.6 yields

$$
\begin{aligned}
& \operatorname{set}_{Q_{P}}[\operatorname{lnl} x]_{\sim_{P}}
\end{aligned}
$$

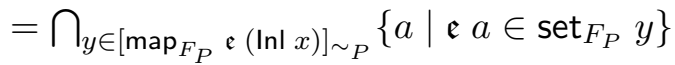

$$
\begin{aligned}
& =\bigcap_{y \in[\operatorname{Inl}(\mathfrak{e} x)]_{\sim_{P}}}\left\{a \mid \mathfrak{e} a \in \operatorname{set}_{F_{P}} y\right\} \\
& =\bigcap_{y \in \operatorname{InI}\langle\text { UNIV }\rangle}\left\{a \mid \mathfrak{e} a \in \operatorname{set}_{F_{P}} y\right\} \\
& =\bigcap_{z \in \operatorname{UNIV}}\left\{a \mid \mathfrak{e} a \in \operatorname{set}_{F_{P}}(\operatorname{In} \mid z)\right\} \\
& =\bigcap_{z \in \operatorname{UNIV}}\{a \mid \mathfrak{e} a=z\} \\
& =\{\}
\end{aligned}
$$

$$
\begin{aligned}
& \operatorname{set}_{Q_{P}}[\operatorname{lnr} x]_{\sim_{P}}
\end{aligned}
$$

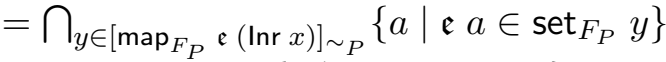

$$
\begin{aligned}
& =\bigcap_{y \in[\operatorname{Inr}(\mathfrak{e} x)]_{\sim_{P}}}\left\{a \mid \mathfrak{e} a \in \operatorname{set}_{F_{P}} y\right\} \\
& =\bigcap_{y \in\{\operatorname{Inr}(\mathfrak{e} x)\}}\left\{a \mid \mathfrak{e} a \in \operatorname{set}_{F_{P}} y\right\} \\
& =\left\{a \mid \mathfrak{e} a \in \operatorname{set}_{F_{P}}(\operatorname{Inr}(\mathfrak{e} x))\right\} \\
& =\{a \mid \mathfrak{e} a=\mathfrak{e} x\} \\
& =\{x\}
\end{aligned}
$$

Next, we express the conditions (3.6) and (3.7) on $Q$ in terms of $\sim$ and $F$. For wide intersections, the condition is as follows:

$$
\forall i . \mathcal{A}_{i} \neq\{\} \wedge\left(\bigcap \mathcal{A}_{i} \neq\{\}\right) \Longrightarrow \bigcap\left\{[\bar{F} \bar{A}]_{\sim} \mid \forall i . A_{i} \in \mathcal{A}_{i}\right\} \subseteq\left[\bigcap\left\{\bar{F} \bar{A} \mid \forall i . A_{i} \in \mathcal{A}_{i}\right\}\right]_{\sim}
$$

The conclusion is as expected: for sets of the form $F \bar{A}$, taking equivalence classes preserves wide intersections. The assumption is the interesting part: preservation is needed only for non-empty intersections. Non-emptiness suffices because Lemma 3.5 relates $F \bar{A}$ to $F \overline{(\{\circledast\} \cup \mathfrak{e}\langle A\rangle)}$ and all intersections of interest therefore contain $\circledast$.

Lemma 3.8. $[F \overline{(\bigcap \mathcal{B})}]_{\sim}=\bigcap\left\{[F \bar{B}]_{\sim} \mid \forall i . B_{i} \in \mathcal{B}_{i}\right\}$ if (3.9) holds for $\overline{\mathcal{A}}$ given by $\mathcal{A}_{i}=\left\{\{\circledast\} \cup \mathfrak{e}\langle B\rangle \mid B \in \mathcal{B}_{i}\right\}$.

Proof. Note that $\overline{\mathcal{A}}$ satisfies the assumption of (3.9). We first show that the other inclusion of (3.9) holds trivially. Let $u \sim x \in \bigcap\left\{F \bar{A} \mid \forall i . A_{i} \in \mathcal{A}_{i}\right\}$. Then $x \in F \bar{A}$ whenever $A_{i} \in \mathcal{A}_{i}$ for all $i$, and so is $u \in[\bar{F} \bar{A}]_{\sim}$. Hence $\bigcap\left\{[\bar{F} \bar{A}]_{\sim} \mid \forall i . A_{i} \in \mathcal{A}_{i}\right\}=$ $\left[\bigcap\left\{F \bar{A} \mid \forall i . A_{i} \in \mathcal{A}_{i}\right\}\right]_{\sim}$.

As $\mathfrak{e}$ is injective and $\circledast$ is not in $\mathfrak{e}$ 's range, we have $\bigcap \mathcal{A}_{i}=\{\circledast\} \cup \mathfrak{e}\left\langle\cap \mathcal{B}_{i}\right\rangle$. We calculate

$$
\begin{aligned}
{[E \overline{(\bigcap \mathcal{B})}]_{\sim} } & =\left[\left\{x \mid\left[\operatorname{map}_{F} \overline{\mathfrak{e}} x\right]_{\sim} \in\left[[F \overline{(\{\circledast\} \cup \mathfrak{e}\langle\bigcap \mathcal{B}\rangle)}]_{\sim}\right\}\right]_{\sim}\right. & & \text { by Lemma } 3.5 \\
& =\left[\left\{x \mid\left[\operatorname{map}_{F} \overline{\mathfrak{e}} x\right]_{\sim} \in[\overline{(\cap \mathcal{A})}]_{\sim}\right\}\right]_{\sim} & & \\
& =\left[\left\{x \mid\left[\operatorname{map}_{F} \overline{\mathfrak{e}} x\right]_{\sim} \in\left[\bigcap\left\{\bar{F} \mid \forall i . A_{i} \in \mathcal{A}_{i}\right\}\right]_{\sim}\right\}\right]_{\sim} & & \text { by }(3.6) \\
& =\left[\left\{x \mid\left[\operatorname{map}_{F} \overline{\mathfrak{e}} x\right]_{\sim} \in \bigcap\left\{[\bar{A}]_{\sim} \mid \forall i . A_{i} \in \mathcal{A}_{i}\right\}\right]_{\sim}\right. & & \text { by the above equality } \\
& =\bigcap\left\{\left[\left\{x \mid\left[\operatorname{map}_{F} \overline{\mathfrak{e}} x\right]_{\sim} \in[\bar{F} \bar{A}]_{\sim}\right\}\right]_{\sim} \mid \forall i . A_{i} \in \mathcal{A}_{i}\right\} & & \\
& =\bigcap\left\{[F \bar{B}]_{\sim} \mid \forall i . B_{i} \in \mathcal{B}_{i}\right\} & & \text { by Lemma } 3.5
\end{aligned}
$$


Condition 3.9 is satisfied trivially for equivalence relations that preserve $\operatorname{set}_{F, i}$, i.e., satisfy (3.3). Examples include permutative structures like finite sets and cyclic lists.

Lemma 3.9. If $\sim$ satisfies (3.3), then $[x]_{\sim} \in[\bar{F} \bar{A}]_{\sim}$ iff $x \in[F \bar{A}$, and condition (3.9) holds.

Proof. Condition (3.3) says that $\operatorname{set}_{F, i} x=\operatorname{set}_{F, i} y$ whenever $x \sim y$. So $[x]_{\sim} \in\left[[F \bar{A}]_{\sim}\right.$ iff $x \in F \bar{A}$ because $F \bar{A}=\left\{x \mid \forall i\right.$ set $\left._{F, i} x \subseteq A_{i}\right\}$ for all $\bar{A}$. Thus, (3.9) holds by the following calculation:

$$
\begin{aligned}
& {[x]_{\sim} \in \bigcap\left\{[F \bar{A}]_{\sim} \mid \forall i . A_{i} \in \mathcal{A}_{i}\right\} \text { iff }[x]_{\sim} \in[\bar{F} \bar{A}]_{\sim} \text { whenever } A_{i} \in \mathcal{A}_{i} \text { for all } i} \\
& \text { iff } x \in \bar{F} \bar{A} \text { whenever } A_{i} \in \mathcal{A}_{i} \text { for all } i \\
& \text { iff } x \in F \overline{(\bigcap \mathcal{A})} \\
& \text { iff }[x]_{\sim} \in[\overline{F \overline{(\bigcap \mathcal{A})}}]_{\sim}
\end{aligned}
$$

In contrast, the non-emptiness assumption is crucial for quotients that identify values with different sets of atoms, such as Example 3.1. In general, such quotients do not preserve empty intersections (Section 5).

We can factor condition (3.9) into a separate property for each type argument $i$ :

$$
\mathcal{A}_{i} \neq\{\} \wedge\left(\bigcap \mathcal{A}_{i}\right) \neq\{\} \Longrightarrow \bigcap_{A \in \mathcal{A}_{i}}\left[\left\{x \mid \operatorname{set}_{F, i} x \subseteq A\right\}\right]_{\sim} \subseteq\left[\left\{x \mid \operatorname{set}_{F, i} x \subseteq \bigcap \mathcal{A}_{i}\right\}\right]_{\sim}
$$

This form is used in our implementation (Section 4). It is arguably more natural to prove for a concrete functor $F$ because each property focuses on a single setter.

Lemma 3.10. Let $\sim$ satisfy (3.1) and (3.2). Then, (3.9) holds iff (3.10) holds for all $i$.

Proof. (3.9) $\Longrightarrow$ (3.10) follows directly by setting $\mathcal{A}_{j}=\{$ UNIV $\}$ for all $j \neq i$, where UNIV is the universe of the respective type. For the other direction, fix $x$ such that for all $\bar{A}$ where $\forall i . A_{i} \in \mathcal{A}_{i}$, there exists $y_{\bar{A}} \in F \bar{A}$ such that $x \sim y_{\bar{A}}$. For every $i$, we have $\operatorname{set}_{F, i} y_{\bar{A}} \subseteq B$ for $B \in \mathcal{A}_{i}$ and hence $x \in \bigcap_{B \in \mathcal{A}_{i}}\left[\left\{x \mid \operatorname{set}_{F, i} x \subseteq B\right\}\right]_{\sim}$. By (3.10) there exists $y_{i}$ such that $\operatorname{set}_{F, i} y_{i} \subseteq \bigcap \mathcal{A}_{i}$ and $x \sim y_{i}$. Fix an arbitrary $a_{i} \in \bigcap \mathcal{A}_{i}$, which exists because $\bigcap \mathcal{A}_{i}$ is assumed to be non-empty. Moreover, set

$$
f_{i} x= \begin{cases}x & \text { if } x \in \bigcap \mathcal{A}_{i} \\ a_{i} & \text { otherwise }\end{cases}
$$

We calculate

$$
\begin{aligned}
x & \sim \operatorname{map}_{F} \overline{\mathrm{id}} y_{1} & & \text { by } x \sim y_{1} \text { and MAP_ID } \\
& =\operatorname{map}_{F} f_{1} \overline{\mathrm{id}} y_{1} & & \text { by } \operatorname{set}_{F, 1} y_{1} \subseteq \bigcap \mathcal{A}_{1} \text { and MAP_CONG } \\
& \sim \operatorname{map}_{F} f_{1} \overline{\mathrm{id}} y_{2} & & \text { by }(3.2) \text { and } y_{1} \sim x \sim y_{2} \\
& =\operatorname{map}_{F} f_{1} f_{2} \overline{\mathrm{id}} y_{2} & & \text { by } \operatorname{set}_{F, 2} y_{2} \subseteq \bigcap \mathcal{A}_{2} \text { and MAP_CONG } \\
& \sim \cdots \sim \operatorname{map}_{F} \bar{f} y_{n} & & \text { similarly } \\
& \sim \operatorname{map}_{F} \bar{f} x & & \text { by } x \sim y_{n} \text { and MAP_CONG }
\end{aligned}
$$

Finally, observe that $\operatorname{map}_{F} \bar{f} x \in \bar{F} \bar{A}$ whenever $\forall i . A_{i} \in \mathcal{A}_{i}$ : We have $\operatorname{set}_{F, i}\left(\operatorname{map}_{F} \bar{f} x\right)=$ $f_{i}\left\langle\operatorname{set}_{F, i} x\right\rangle \subseteq \bigcap \mathcal{A}_{i} \subseteq A_{i}$ using SET_MAP and the definition of $f_{i}$.

Many functors in practice contain only finitely many elements, i.e., $\operatorname{set}_{F, i} x$ is always finite. This includes all inductive datatypes built only from sums and products, e.g., finite lists and finitely branching trees. Condition (3.10) is always satisfied for such functors, 
because wide intersections boil down to finite intersections in this case and Trnková [Trn69] showed that all Set functors preserve non-empty binary intersections.

Lemma 3.11. If $\operatorname{set}_{F, i} x$ is finite for all $x$, then (3.10) holds for all equivalence relations $\sim$ that satisfy (3.2).

Proof. Fix $a_{0} \in \bigcap \mathcal{A}_{i}$ and let $x \in \bigcap_{A \in \mathcal{A}_{i}}\left[\left\{x \mid \operatorname{set}_{F, i} x \subseteq A\right\}\right]_{\sim}$. So for $A \in \mathcal{A}_{i}$, there exists a $y_{A}$ such that $x \sim y_{A}$ and $\operatorname{set}_{F, i} y_{A} \subseteq A$. Set $B_{A}=\operatorname{set}_{F, i} y_{A} \cup\left\{a_{0}\right\}$ and $\mathcal{B}=\left\{B_{A} \mid A \in \mathcal{A}_{i}\right\}$. Then $\bigcap \mathcal{B} \subseteq \bigcap \mathcal{A}_{i}$. Since all $B_{A}$ are finite, there exist finitely many $A_{j}(j=1, \ldots, n$ for some $n$ ) such that $\bigcap_{j=1}^{n} B_{A_{j}} \subseteq \bigcap \mathcal{B}$; for example, pick $A_{1} \in \mathcal{A}_{i}$ arbitrarily, let $B_{A_{1}}-\cap \mathcal{B}=$ $\left\{b_{2}, \ldots, b_{n}\right\}$, and choose $A_{j} \in \mathcal{A}_{i}$ such that $b_{j} \notin B_{A_{j}}$ for $j=2, \ldots, n$. Clearly, $a_{0} \in B_{A_{j}}$ for all $j$ by construction.

It suffices to show that there exists a $z$ such that $x \sim z$ and $\operatorname{set}_{F, i} z \subseteq \bigcap_{j=1}^{n} B_{A_{j}}$. This $z$ proves that $x \in\left[\left\{x \mid \operatorname{set}_{F, i} x \subseteq \bigcap \mathcal{A}_{i}\right\}\right]_{\sim}$ because $\bigcap_{j=1}^{n} B_{A_{j}} \subseteq \bigcap \mathcal{B} \subseteq \bigcap \mathcal{A}_{i}$. We show the existence of such a $z$ by induction over the finitely many $A_{j}$. If there is only a single $A_{1}$, i.e., $n=1$, then choose $z=y_{A_{1}}$. Otherwise, let $z^{\prime}$ be such that $x \sim z^{\prime}$ and $\operatorname{set}_{F, i} z^{\prime} \subseteq \bigcap_{j=1}^{n-1} B_{A_{j}}$ by the induction hypothesis. Define $f_{i}(a)=a$ for $a \in B_{A_{n}}$ and $f_{i}(a)=a_{0}$ for $a \notin B_{A_{n}}$. Set $f_{i^{\prime}}=\mathrm{id}$ for $i^{\prime} \neq i$ and choose $z=\operatorname{map}_{F} \bar{f} z^{\prime}$. Then, $z \sim \operatorname{map}_{F} \bar{f} y_{A_{n}}$ follows from $z^{\prime} \sim x \sim y_{A_{n}}$ by (3.2) and $\operatorname{map}_{F} \bar{f} y_{A_{n}}=\operatorname{map}_{F} \overline{\text { id }} y_{A_{n}}=y_{A_{n}}$ holds by MAP_CONG and MAP_ID, so $z \sim y_{A_{n}} \sim x$. Moreover, SET_MAP gives $\operatorname{set}_{F, i} z=f_{i}\left\langle\operatorname{set}_{F, i} z^{\prime}\right\rangle \subseteq f_{i}\left\langle\bigcap_{j=1}^{n-1} B_{A_{j}}\right\rangle \subseteq$ $\left(\bigcap_{j=1}^{n-1} B_{A_{j}}\right) \cap B_{A_{n}}=\bigcap_{j=1}^{n} B_{A_{j}}$.

Preservation of preimages amounts to the following unsurprising condition:

$$
\forall i . f_{i}^{-1}\left\langle A_{i}\right\rangle \neq\{\} \Longrightarrow\left(\operatorname{map}_{F} \bar{f}\right)^{-1}\left\langle\bigcup[\bar{F} \bar{A}]_{\sim}\right\rangle \subseteq \bigcup\left[\left(\operatorname{map}_{F} \bar{f}\right)^{-1}\langle[F \bar{A}\rangle]_{\sim}\right.
$$

As for wide intersections, taking equivalence classes must preserve non-empty preimages. Again, non-emptiness comes from $\circledast$ being contained in all sets of interest.

Lemma 3.12. $E \overline{\left(g^{-1}\langle B\rangle\right)}=\left(\operatorname{map}_{F} \bar{g}\right)^{-1}\langle E \bar{B}\rangle$ if (3.11) is satisfied for $\bar{A}$ and $\bar{f}$ given by $A_{i}=\{\circledast\} \cup \mathfrak{e}\left\langle B_{i}\right\rangle$ and $f_{i}=\operatorname{map}_{1+} g_{i}$.

Proof. We have $f_{i}^{-1}\left\langle A_{i}\right\rangle=\{\circledast\} \cup \mathfrak{e}\left\langle g_{i}^{-1}\left\langle B_{i}\right\rangle\right\rangle$ and the precondition of (3.11) holds. The inclusion from right to left holds trivially: If $x \in \bigcup\left[\left(\operatorname{map}_{F} \bar{f}\right)^{-1}\langle\bar{F} \bar{A}\rangle\right]_{\sim}$, then there exists a $y$ such that $x \sim y$ and $\operatorname{map}_{F} \bar{f} y \in E A$; so $\operatorname{map}_{F} \bar{f} x \sim \operatorname{map}_{F} \bar{f} y$ by (3.2). We calculate

$$
\begin{aligned}
& \left.x \in E \overline{\left(g^{-1}\langle B\rangle\right)} \longleftrightarrow\left[\operatorname{map}_{F} \overline{\mathfrak{e}} x\right]_{\sim} \in\left[\overline{\left(F \overline{(\{\otimes} \cup \mathfrak{e}\left\langle g^{-1}\langle B\rangle\right\rangle\right)}\right]_{\sim}\right\} \quad \text { by Lemma } 3.5 \\
& \longleftrightarrow\left[\operatorname{map}_{F} \overline{\mathfrak{e}} x\right]_{\sim} \in\left[\bar{F} \overline{\left(f^{-1}\langle A\rangle\right)}\right]_{\sim} \quad \text { by choice of } \bar{f} \\
& \longleftrightarrow\left[\operatorname{map}_{F} \overline{\mathfrak{e}} x\right]_{\sim} \in\left[\left(\operatorname{map}_{F} \bar{f}\right)^{-1}\langle\bar{F} \bar{A}\rangle\right]_{\sim} \quad \text { by }(3.7) \\
& \longleftrightarrow \operatorname{map}_{F} \overline{\mathfrak{e}} x \in \bigcup\left[\left(\operatorname{map}_{F} \bar{f}\right)^{-1}\langle F \bar{A}\rangle\right]_{\sim} \\
& \longleftrightarrow \operatorname{map}_{F} \overline{\mathfrak{e}} x \in\left(\operatorname{map}_{F} \bar{f}\right)^{-1}\left\langle\bigcup\left[[F \bar{A}]_{\sim}\right\rangle \quad\right. \text { by (3.11) } \\
& \longleftrightarrow\left[\operatorname{map}_{F} \bar{f}\left(\operatorname{map}_{F} \overline{\mathfrak{e}} x\right)\right]_{\sim} \in[\bar{F} \bar{A}]_{\sim} \\
& \longleftrightarrow\left[\operatorname{map}_{F} \overline{\mathfrak{e}}\left(\operatorname{map}_{F} \bar{g} x\right)\right]_{\sim} \in\left[[\bar{F} \bar{A}]_{\sim} \quad \text { as } f_{i} \circ \mathfrak{e}=\mathfrak{e} \circ g_{i}\right. \\
& \longleftrightarrow x \in\left(\operatorname{map}_{F} \bar{g}\right)^{-1}\langle F \bar{B}\rangle \quad \text { by Lemma } 3.5
\end{aligned}
$$

We do not elaborate on how to establish preimage preservation (3.11) any further as it follows from subdistributivity, which we will look at in the next subsection. Instead, we show that the quotient setters $\operatorname{set}_{Q}$ are natural transformations under conditions (3.9) and (3.11). 
Lemma 3.13. Under the conditions (3.9) and (3.11), set Q $_{, i}$ is a natural transformation, i.e., $\operatorname{set}_{Q, i}\left(\operatorname{map}_{Q} \bar{f}[x]_{\sim}\right)=f_{i}\left\langle\operatorname{set}_{Q, i}[x]_{\sim}\right\rangle$, and therefore $\operatorname{set}_{Q, i}\left[\operatorname{map}_{F} \bar{f} x\right]_{\sim}=f_{i}\left\langle\operatorname{set}_{Q, i}[x]_{\sim}\right\rangle$.

Proof. By Lemma 3.8, $Q$ preserves wide intersections (3.6). Similarly, Lemma 3.12 shows that $Q$ preserves preimages (3.7). The claim follows with [Gum05, Theorem 6].

Lemma 3.14. Under the conditions (3.9) and (3.11), $\operatorname{map}_{F} \bar{f} x \sim \operatorname{map}_{F} \bar{g} x$ if $f_{i} z=g_{i} z$ for all $i$ and all $z \in \operatorname{set}_{Q, i}[x]_{\sim}$.

Proof. We first show that $[x]_{\sim} \in Q \overline{\left(\operatorname{set}_{Q}[x]_{\sim}\right)}$. We observe

$$
\begin{aligned}
& {[x]_{\sim} \in Q \overline{\left(\operatorname{set}_{Q}[x]_{\sim}\right)} \longleftrightarrow[x]_{\sim} \in Q \overline{\left(\bigcap_{y \in[\operatorname{map} \overline{\mathfrak{e}} x]_{\sim}}\left\{a \mid \mathfrak{e} a \in \operatorname{set}_{F} y\right\}\right)} \quad \text { by Theorem } 3.6} \\
& \longleftrightarrow[x]_{\sim} \in \bigcap_{y \in[\operatorname{map} \overline{\mathfrak{e}} x]_{\sim}}\left[F \overline{\left\{a \mid \mathfrak{e} a \in \operatorname{set}_{F} y\right\}}\right]_{\sim} \quad \text { by Lemma } 3.8 \\
& \longleftrightarrow \forall y \in[\operatorname{map} \overline{\mathfrak{e}} x]_{\sim} \cdot[x]_{\sim} \in\left[F \overline{\left\{a \mid \mathfrak{e} a \in \operatorname{set}_{F} y\right\}}\right]_{\sim}
\end{aligned}
$$

So let $y \sim \operatorname{map} \overline{\mathfrak{e}} x$. Then $y \in F \overline{\left(\{\circledast\} \cup \operatorname{set}_{F} y\right)}$ by definition of $F$ and thus $[x]_{\sim} \in$ $\left[F \overline{\left\{a \mid \mathfrak{e} a \in \operatorname{set}_{F} y\right\}}\right]_{\sim}$ by Lemma 3.5 using $\mathfrak{e}\left\langle\left\{a \mid \mathfrak{e} a \in \operatorname{set}_{F, i} y\right\}\right\rangle=\operatorname{set}_{F, i} y$ for all $i$.

By (3.8), we therefore have $\operatorname{map}_{F} \overline{\mathfrak{e}} x \sim \operatorname{map}_{F} \bar{h} x$ for $h_{i} a=\mathfrak{e} a$ for $a \in \operatorname{set}_{Q, i}$ and $h_{i} a=\circledast$ otherwise. Then

$$
\begin{aligned}
\operatorname{map}_{F} \bar{f} x & =\operatorname{map}_{F} \overline{\mathfrak{e}^{-1}}\left(\operatorname{map}_{F} \overline{\left(\operatorname{map}_{1+f}\right)}\left(\operatorname{map}_{F} \overline{\mathfrak{e}} x\right)\right) & & \text { as } \mathfrak{e}^{-1} \circ \operatorname{map}_{1+} f_{i} \circ \mathfrak{e}=f_{i} \\
& \sim \operatorname{map}_{F} \overline{\mathfrak{e}^{-1}}\left(\operatorname{map}_{F} \overline{\left(\operatorname{map}_{1+} f\right)}\left(\operatorname{map}_{F} \bar{h} x\right)\right) & & \text { by }(3.2) \\
& =\operatorname{map}_{F} \overline{\mathfrak{e}^{-1}}\left(\operatorname{map}_{F} \overline{\left(\operatorname{map}_{1+} g\right)}\left(\operatorname{map}_{F} \bar{h} x\right)\right) & & \text { by MAP_CONG } \\
& \sim \operatorname{map}_{F} \overline{\mathfrak{e}^{-1}}\left(\operatorname{map}_{F} \overline{\left(\operatorname{map}_{1+} g\right)}\left(\operatorname{map}_{F} \mathfrak{e} x\right)\right) & & \text { by }(3.2) \\
& =\operatorname{map}_{F} \bar{g} x & & \text { as } \mathfrak{e}^{-1} \circ \operatorname{map}_{1+} g_{i} \circ \mathfrak{e}=g_{i}
\end{aligned}
$$

3.3. The quotient's relator. In the previous section, we have shown that it is not a good idea to naively lift the setter and a more general construction is needed. We now show that the same holds for the relator. The following straightforward definition

$$
\text { lift_definition } \operatorname{rel}_{Q}:: \overline{(\alpha \otimes \beta)} \rightarrow \bar{\alpha} Q \otimes \bar{\beta} Q \text { is } \text { rel }_{F}
$$

relates two equivalence classes $[x]_{\sim}$ and $[y]_{\sim}$ iff there are representatives $x^{\prime} \in[x]_{\sim}$ and $y^{\prime} \in[y]_{\sim}$ such that $\left(x^{\prime}, y^{\prime}\right) \in \operatorname{rel}_{F} \bar{R}$. This relator does not satisfy IN_REL in general.

Example 3.15 (Example 3.7 continued). By the lifted definition, $\left([\ln \mid x]_{\sim_{P}},[\ln \mid y]_{\sim_{P}}\right) \notin$ rel $_{Q_{P}}\{\}$ because there are no $\left(x^{\prime}, y^{\prime}\right)$ in the empty relation \{\} that could be used to relate using rel $_{F_{P}}$ the representatives $\operatorname{Inl} x^{\prime}$ and $\operatorname{Inl} y^{\prime}$. However, the witness $z=[\operatorname{Inl}(x, y)]_{\sim_{P}}$ satisfies the right-hand side of IN_REL as $Q\{\}=\left\{[\operatorname{lnl}]_{\sim_{P}}\right\}$.

So what is the relationship between rel $_{Q}$ and rel $_{F}$ and under what conditions does the subdistributivity property REL_COMP hold? Like for the setter, we avoid the problematic case of empty relations by switching to $1+\alpha$. The relator rel $1+$ adds the pair $(\circledast, \circledast)$ to every relation $R$ and thereby ensures that all relations and their compositions are non-empty. Accordingly, we obtain the following characterization under the conditions (3.9) and (3.11):

Theorem 3.16 (Relator characterization).

$$
\left([x]_{\sim},[y]_{\sim}\right) \in \operatorname{rel}_{Q} \bar{R} \longleftrightarrow\left(\operatorname{map}_{F} \overline{\mathfrak{e}} x, \operatorname{map}_{F} \overline{\mathfrak{e}} y\right) \in\left(\sim \bullet \operatorname{rel}_{F} \overline{\left(r e l_{1+} R\right)} \bullet \sim\right)
$$


Proof. Applying IN_REL to both rel $_{Q}$ and $\mathrm{rel}_{F}$, it suffices to show that

$$
\exists z .\left(\forall i . \operatorname{set}_{Q, i}[z]_{\sim} \subseteq R_{i}\right) \wedge \operatorname{map}_{F} \overline{\text { fst }} z \sim x \wedge \operatorname{map}_{F} \overline{\text { snd }} z \sim y
$$

if and only if

$$
\exists z^{\prime} .\left(\forall i . \operatorname{set}_{F, i} z^{\prime} \subseteq \operatorname{rel}_{1+} R_{i}\right) \wedge \operatorname{map}_{F} \overline{\text { fst }} z^{\prime} \sim \operatorname{map}_{F} \overline{\mathfrak{e}} x \wedge \operatorname{map}_{F} \overline{\operatorname{snd}} z^{\prime} \sim \operatorname{map}_{F} \overline{\mathfrak{e}} y .
$$

Specifically, we show how to convert the witnesses $z$ and $z^{\prime}$.

From (3.12) to (3.13): Let the function $f_{i}$ send the pair $(a, b)$ to $(\mathfrak{e} a, \mathfrak{e} b)$ if $(a, b) \in R_{i}$ and otherwise to $(\circledast, \circledast)$. We choose $z^{\prime}=\operatorname{map}_{F} \bar{f} z$ and prove that it is a witness for (3.13). By SET_MAP we have $\operatorname{set}_{F, i} z^{\prime}=f_{i}\left\langle\operatorname{set}_{F, i} z\right\rangle$. The image of any set under $f_{i}$ is clearly included in rel $1+R_{i}$, hence $\operatorname{set}_{F, i} z^{\prime} \subseteq$ rel $_{1+} R_{i}$ for all $i$. Next, we calculate

$$
\begin{aligned}
\operatorname{map}_{F} \overline{\mathrm{fst}} z^{\prime} & =\operatorname{map}_{F} \overline{(\mathrm{fst} \circ f)} z & & \text { by MAP_COMP } \\
& \sim \operatorname{map}_{F} \overline{(\mathfrak{e} \circ \mathrm{fst})} z & & \text { by Lemma } 3.14 \text { and } \forall i . \operatorname{set}_{Q, i}[z]_{\sim} \subseteq R_{i} \\
& =\operatorname{map}_{F} \overline{\mathfrak{e}}\left(\operatorname{map}_{F} \overline{\text { fst }} z\right) & & \text { by MAP_COMP } \\
& \sim \operatorname{map}_{F} \overline{\bar{e}} x & & \text { by }(3.2) \text { and } \operatorname{map}_{F} \overline{\text { fst }} z=x .
\end{aligned}
$$

The third conjunct $\operatorname{map}_{F} \overline{\text { snd }} z^{\prime} \sim \operatorname{map}_{F} \overline{\mathfrak{e}} y$ is derived similarly.

From (3.13) to (3.12): Note that $\operatorname{set}_{Q, i}\left[\operatorname{map}_{F} \overline{\mathrm{fst}} z^{\prime}\right]_{\sim}=\operatorname{set}_{Q, i}\left[\operatorname{map}_{F} \overline{\mathfrak{e}} x\right]_{\sim}$ as $\operatorname{map}_{F} \overline{\mathrm{fst}} z^{\prime} \sim \operatorname{map}_{F} \overline{\mathfrak{e}} x$ are in the same equivalence class. Using Lemma 3.13 twice it follows that fst $\left\langle\operatorname{set}_{Q, i}\left[z^{\prime}\right]_{\sim}\right\rangle=\mathfrak{e}\left\langle\operatorname{set}_{Q, i}[x]_{\sim}\right\rangle$. A similar observation can be made about the second projection and $y$. Therefore, $\operatorname{set}_{Q, i}\left[z^{\prime}\right]_{\sim}$ consists only of pairs of the form $(\mathfrak{e} a, \mathfrak{e} b)$. Accordingly, we use a function $g$ that maps $\mathfrak{e} a$ to $a$ and $\circledast$ to some unspecified value. Then choose $z=\operatorname{map}_{F} \overline{(g \times g)} z^{\prime}$ as the witness for (3.12), where $g \times g$ denotes the componentwise application of $g$ to pairs. We have

$$
\begin{aligned}
\operatorname{set}_{Q, i}[z]_{\sim} & =(g \times g)\left\langle\operatorname{set}_{Q, i}\left[z^{\prime}\right]_{\sim}\right\rangle & & \text { by Lemma } 3.13 \\
& =(\mathfrak{e} \times \mathfrak{e})^{-1}\left\langle\operatorname{set}_{Q, i}\left[z^{\prime}\right]_{\sim}\right\rangle & & \text { by the above observations } \\
& \subseteq(\mathfrak{e} \times \mathfrak{e})^{-1}\left\langle\operatorname{set}_{F, i} z^{\prime}\right\rangle & & \text { by Theorem } 3.6 \\
& \subseteq(\mathfrak{e} \times \mathfrak{e})^{-1}\left\langle\operatorname{ret}_{1+} R_{i}\right\rangle & & \text { by assumption and monotonicity of preimage } \\
& =R_{i} . & &
\end{aligned}
$$

Moreover, $\operatorname{map}_{F} \overline{\mathrm{fst}} z=\operatorname{map}_{F} \overline{(g \circ \mathrm{fst})} z^{\prime} \sim \operatorname{map}_{F} \overline{(g \circ \mathfrak{e})} x=x$ by MAP_COMP, (3.2) applied to $\operatorname{map}_{F} \overline{\text { fst }} z^{\prime} \sim \operatorname{map}_{F} \overline{\mathfrak{e}} x$, and MAP_ID; analogously for $\operatorname{map}_{F} \overline{\text { snd }} z \sim y$.

Example 3.17 (Example 3.15 continued). For arbitrary $x, y$, and $R$, we have

$$
\begin{aligned}
& \left([\ln x]_{\sim_{P}},[\operatorname{Inl} y]_{\sim_{P}}\right) \in \operatorname{rel}_{Q} R \\
\longleftrightarrow & \left(\operatorname{map}_{F_{P}} \mathfrak{e}(\operatorname{Inl} x), \operatorname{map}_{F_{P}} \mathfrak{e}(\operatorname{Inl} y)\right) \in\left(\sim_{P} \bullet \operatorname{rel}_{F_{P}}\left(\operatorname{rel}_{1+} R\right) \bullet \sim_{P}\right) \\
\longleftrightarrow & \left(\exists x^{\prime} y^{\prime} . \operatorname{Inl}(\mathfrak{e} x) \sim_{P} x^{\prime} \wedge\left(x^{\prime}, y^{\prime}\right) \in \operatorname{rel}_{F_{P}}\left(\operatorname{rel}_{1+} R\right) \wedge y^{\prime} \sim_{P} \operatorname{Inl}(\mathfrak{e} y)\right) \\
\longleftrightarrow & \left(\exists x^{\prime \prime} y^{\prime \prime} .\left(\operatorname{Inl} x^{\prime \prime}, \operatorname{Inl} y^{\prime \prime}\right) \in \operatorname{rel}_{F_{P}}\left(\operatorname{rel}_{1+} R\right)\right) \\
\longleftrightarrow & \left(\exists x^{\prime \prime} y^{\prime \prime} .\left(x^{\prime \prime}, y^{\prime \prime}\right) \in \operatorname{rel}_{1+} R\right),
\end{aligned}
$$

which is always true since $(\circledast, \circledast) \in$ rel $_{1+} R$. On the other hand,

$$
\begin{aligned}
& \left([\operatorname{Inr} x]_{\sim_{P}},[\operatorname{Inr} y]_{\sim_{P}}\right) \in \operatorname{rel}_{Q} R \\
\longleftrightarrow & \left(\operatorname{map}_{F_{P}} \mathfrak{e}(\operatorname{Inr} x), \operatorname{map}_{F_{P}} \mathfrak{e}(\operatorname{Inr} y)\right) \in\left(\sim_{P} \bullet \operatorname{rel}_{F_{P}}\left(\operatorname{rel}_{1+} R\right) \bullet \sim_{P}\right) \\
\longleftrightarrow & \left(\exists x^{\prime} y^{\prime} . \ln (\mathfrak{e} x) \sim_{P} x^{\prime} \wedge\left(x^{\prime}, y^{\prime}\right) \in \operatorname{rel}_{F_{P}}\left(\operatorname{rel}_{1+} R\right) \wedge y^{\prime} \sim_{P} \operatorname{Inr}(\mathfrak{e} y)\right) \\
\longleftrightarrow & (\operatorname{Inr}(\mathfrak{e} x), \operatorname{Inr}(\mathfrak{e} y)) \in \operatorname{rel}_{F_{P}}\left(\operatorname{rel}_{1+} R\right) \\
\longleftrightarrow & (\mathfrak{e} x, \mathfrak{e} y) \in \operatorname{rel} l_{1+} R \\
\longleftrightarrow & (x, y) \in R .
\end{aligned}
$$


It is easy to see that $\mathrm{rel}_{Q} R$ does not relate $[\operatorname{lnl} x]_{\sim_{P}}$ and $[\operatorname{lnr} y]_{\sim_{P}}$ or vice versa. Therefore, rel $_{Q}$ behaves exactly like the relator of $1+\alpha$, as expected.

Moreover, the following condition on $\sim$ characterizes when rel $_{Q}$ satisfies REL_COMP. Again, the non-emptiness assumptions for $R_{i} \bullet S_{i}$ come from rel ${ }_{1+}$ extending any relation $R$ with the pair $(\circledast, \circledast)$.

$$
\left(\forall i . R_{i} \bullet S_{i} \neq\{\}\right) \Longrightarrow \operatorname{rel}_{F} \bar{R} \bullet \sim \bullet \operatorname{rel}_{F} \bar{S} \subseteq \sim \bullet \operatorname{rel}_{F} \overline{(R \bullet S)} \bullet \sim
$$

It turns out that this condition implies the respectfulness of the mapper (3.2). Intuitively, the relator is a generalization of the mapper. Furthermore, it is well known that subdistributivity implies preimage preservation [GS05]. Since our conditions on $\sim$ characterize these preservation properties, it is no surprise that the latter implication carries over.

Lemma 3.18. For an equivalence relation $\sim$, condition (3.14) implies respectfulness (3.2) and preimage preservation (3.11).

Proof. To show (3.2), fix $x$ and $y$ such that $x \sim y$. Choose the relations $R_{i}=\left\{\left(f_{i} a, a\right) \mid\right.$ True $\}$ and $S_{i}=\left\{\left(a, f_{i} a\right) \mid\right.$ True $\}$. Then $R_{i} \bullet S_{i} \neq\{\}$ because types in HOL are non-empty and so is $f_{i}$ 's image. We have $\left(\operatorname{map}_{F} \bar{f} x, x\right) \in \operatorname{rel}_{F} \bar{R}$ and $\left(y, \operatorname{map}_{F} \bar{f} y\right) \in \operatorname{rel}_{F} \bar{S}$ by well-known BNF properties. Therefore, $\left(\operatorname{map}_{F} \bar{f} x, \operatorname{map}_{F} \bar{f} y\right) \in\left(\operatorname{rel}_{F} \bar{R} \bullet \sim \bullet \operatorname{rel}_{F} \bar{S}\right)$. Using (3.14), there exist $z$ and $z^{\prime}$ such that $\operatorname{map}_{F} \bar{f} x \sim z,\left(z, z^{\prime}\right) \in \operatorname{rel}_{F} \overline{(R \bullet S)}$, and $z^{\prime} \sim \operatorname{map}_{F} \bar{f} y$. Note that $R_{i} \bullet S_{i}$ is equality restricted to $f_{i}$ 's image. This implies $z=z^{\prime}$, again by the BNF properties. Thus $\operatorname{map}_{F} \bar{f} x \sim \operatorname{map}_{F} \bar{f} y$.

It remains to prove (3.11). Let $x$ and $y$ be such that $\operatorname{map}_{F} \bar{f} x \sim y$ and $y \in F \bar{A}$. Choose $R_{i}=A_{i} \times A_{i}$ and $S_{i}=\left\{\left(f_{i} a, a\right) \mid\right.$ True $\}$. Then $R_{i} \bullet S_{i} \neq\{\}$ as $f_{i}^{-1}\left\langle A_{i}\right\rangle \neq\{\}$ by assumption in (3.11). Moreover, $(y, y) \in \operatorname{rel}_{F} \bar{R}$ as $\operatorname{map}_{F} \overline{(\lambda a .(a, a))} y \in F \bar{R}$. Further, $\left(\operatorname{map}_{F} \bar{f} x, x\right) \in$ $\operatorname{rel}_{F} \bar{S}$ as $\operatorname{map}_{F} \overline{(\lambda a .(f a, a))} x \in E \bar{S}$. Therefore, $(y, x) \in \sim \bullet \operatorname{rel}_{F} \overline{(R \bullet S)} \bullet \sim$ by (3.14). So there are $u$ and $v$ such that $y \sim u,(u, v) \in \operatorname{rel}_{F} \overline{(R \bullet S)}$, and $v \sim x$. By the BNF properties, there is a $w$ such that $(u, w) \in \operatorname{rel}_{F} \bar{R}$ and $(w, v) \in \operatorname{rel}_{F} \bar{S}$. Then, $w \in F \bar{A}$ and $v=\operatorname{map}_{F} \bar{f} w$. So $v \in\left(\operatorname{map}_{F} \bar{f}\right)^{-1}\langle\bar{F} \bar{A}\rangle$ and $x \sim v$.

In summary, we obtain the following main preservation theorem:

Theorem 3.19. The quotient $\bar{\alpha} Q=\bar{\alpha} F / \sim$ inherits the structure from the BNF $\bar{\alpha} F$ with the mapper $\operatorname{map}_{Q} \bar{f}[x]_{\sim}=\left[\operatorname{map}_{F} \bar{f} x\right]_{\sim}$ if $\sim$ satisfies the conditions (3.1), (3.9), and (3.14). The setters and relator are given by Theorems 3.6 and 3.16, respectively.

Example 3.20. A terminated coinductive list $(\alpha, \beta)$ tllist is either a finite list of $\alpha$ values terminated by a single $\beta$ value, or an infinite list of $\alpha$ values. This type can be seen as a quotient of pairs $\alpha$ llist $\times \beta$, where the first component stores the possibly infinite list given by a codatatype llist and the second component stores the terminator. The equivalence relation identifies all pairs with the same infinite list in the first component, effectively removing the terminator from infinite lists. ${ }^{3}$ Let $(x s, b) \sim_{t l l i s t}(y s, c)$ iff $x s=y s$ and, if $x s$ is finite, $b=c$. Like $\sim_{P}$ from Example 3.1, $\sim_{\text {tllist }}$ does not satisfy the naive condition (3.3).

$$
\begin{aligned}
& \text { codatatype } \alpha \text { llist }=\mathrm{LNil} \mid \text { LCons } \alpha(\alpha \text { llist }) \\
& \text { quotient_type }(\alpha, \beta) \text { tllist }=(\alpha \text { llist } \times \beta) / \sim_{\text {tllist }}
\end{aligned}
$$

\footnotetext{
${ }^{3}$ Clearly, tllist could be defined directly as a codatatype. When Isabelle had no codatatype command, one of the authors formalized tllist via this quotient [Loc10, version for Isabelle2013].
} 
Our goal is the construction of (co)datatypes with recursion through quotients such as $(\alpha, \beta)$ tllist. As a realistic example, consider an inductive model of a finite interactive system that produces a possibly unbounded sequence of outputs out for every input in:

$$
\text { datatype system }=\operatorname{Step~}(\text { in } \rightarrow(\text { out, system }) \text { tllist })
$$

This datatype definition is only possible if tllist is a BNF in $\beta$. Previously, this had to be shown by manually defining the mapper and setters and proving the BNF properties. Theorem 3.19 identifies the conditions under which tllist inherits the BNF structure of its underlying type, and it allows us to automate these definitions and proofs. For tlllist, the conditions can be discharged easily using automatic proof methods and a simple lemma about llist's relator that states that related lists are either both finite or infinite.

3.4. Subdistributivity via confluent relations. Among the BNF properties, subdistributivity (REL_COMP) is typically the hardest to show. For example, distinct lists (type $\alpha$ dlist) have been shown to be a BNF. The manual proof requires 126 lines. Of these, the subdistributivity proof takes about 100 lines. Yet, with the theory developed so far, essentially the same argument is needed for the subdistributivity condition (3.14). We now present a sufficient criterion for subdistributivity that simplifies such proofs. For dlist, this shortens the subdistributivity proof to 58 lines. With our lift_bnf command (Section 4), the whole proof is now 64 lines, half of the manual proof.

Equivalence relations are often (or can be) expressed as the equivalence closure of a rewrite relation $\rightsquigarrow$. For example, the subdistributivity proof for distinct lists views $\alpha$ dlist as the quotient $\alpha$ list $/ \sim_{\text {dlist }}$ with $x s \sim_{\text {dlist }} y s$ iff remdups $x s=$ remdups $y s$, where remdups $x s$ keeps only the last occurrence of every element in $x s$. So, $\sim$ dlist is the equivalence closure of the following relation $\rightsquigarrow d l i s t$, where $\cdot$ concatenates two lists:

$$
x s \cdot[x] \cdot y s \rightsquigarrow \text { dlist } x s \cdot y s \text { if } x \in \text { set } y s
$$

We use the following notation: « denotes the reverse relation, i.e., $x \rightsquigarrow y$ iff $y \rightsquigarrow x$. Further, $\stackrel{*}{\rightsquigarrow}$ denotes the reflexive and transitive closure, and $\stackrel{*}{\rightsquigarrow}$ the equivalence closure. A relation $\rightsquigarrow$ is confluent iff whenever $x \stackrel{*}{\rightsquigarrow} y$ and $x \stackrel{*}{\rightsquigarrow} z$, then there exists a $u$ such that $y \stackrel{*}{\rightsquigarrow} u$ and $z \stackrel{*}{\rightsquigarrow} u$-or, equivalently in pointfree style, if $(\stackrel{*}{\sim} \bullet \stackrel{*}{\rightsquigarrow}) \subseteq(\stackrel{*}{\rightsquigarrow} \bullet \stackrel{*}{\sim})$.

Theorem 3.21 (Subdistributivity via confluent relations). Let an equivalence relation satisfy (3.2). Then, it also satisfies (3.14) if there is a confluent relation $\rightsquigarrow$ with the following properties:

(i) The equivalence relation is contained in $\rightsquigarrow$ 's equivalence closure: $(\sim) \subseteq(\stackrel{*}{\sim}){ }^{4}$

(ii) The relation factors through projections: if $\operatorname{map}_{F} \overline{f_{s t}} x \rightsquigarrow y$ then there exists a $y^{\prime}$ such that $y=\operatorname{map}_{F} \overline{\text { fst }} y^{\prime}$ and $x \sim y^{\prime}$ and $\operatorname{set}_{F, i} y^{\prime} \subseteq \operatorname{set}_{F, i} x$ for all $i$; and similarly for snd.

Proof. The proof is illustrated in Fig. 1. The proof starts at the top with $(x, z) \in\left(\operatorname{rel}_{F} \bar{R} \bullet\right.$ $\left.\sim \operatorname{rel}_{F} \bar{S}\right)$, i.e., there are $y$ and $y^{\prime}$ such that $(x, y) \in \operatorname{rel}_{F} \bar{R}$ and $y \sim y^{\prime}$ and $\left(y^{\prime}, z\right) \in \operatorname{rel}_{F} \bar{S}$. We show $(x, z) \in\left(\sim \bullet \operatorname{rel}_{F} \overline{(R \bullet S)} \bullet \sim\right)$ by establishing the path from $x$ to $z$ via $x^{\prime}$ and $z^{\prime}$ along the three other borders of the diagram.

\footnotetext{
${ }^{4}$ The other inclusion $(\stackrel{*}{*}) \subseteq(\sim)$ follows from the second condition with $(3.2)$ : For $x \rightsquigarrow y$, let $x^{\prime}=$ $\operatorname{map}_{F} \overline{(\lambda a .(a, a))} x$. As $x=\operatorname{map}_{F} \overline{\text { fst }} x^{\prime}$, there exists a $y^{\prime}$ with $x^{\prime} \sim y^{\prime}$ and $y=\operatorname{map}_{F} \overline{\text { fst }} y^{\prime}$. Applying (3.2) to $x^{\prime} \sim y^{\prime}$, we have $x=\operatorname{map}_{F} \overline{\text { fst }} x^{\prime} \sim \operatorname{map}_{F} \overline{\text { fst }} y^{\prime}=y$. So $(\rightsquigarrow) \subseteq(\sim)$ and therefore $(\stackrel{*}{*}) \subseteq(\sim)$.
} 


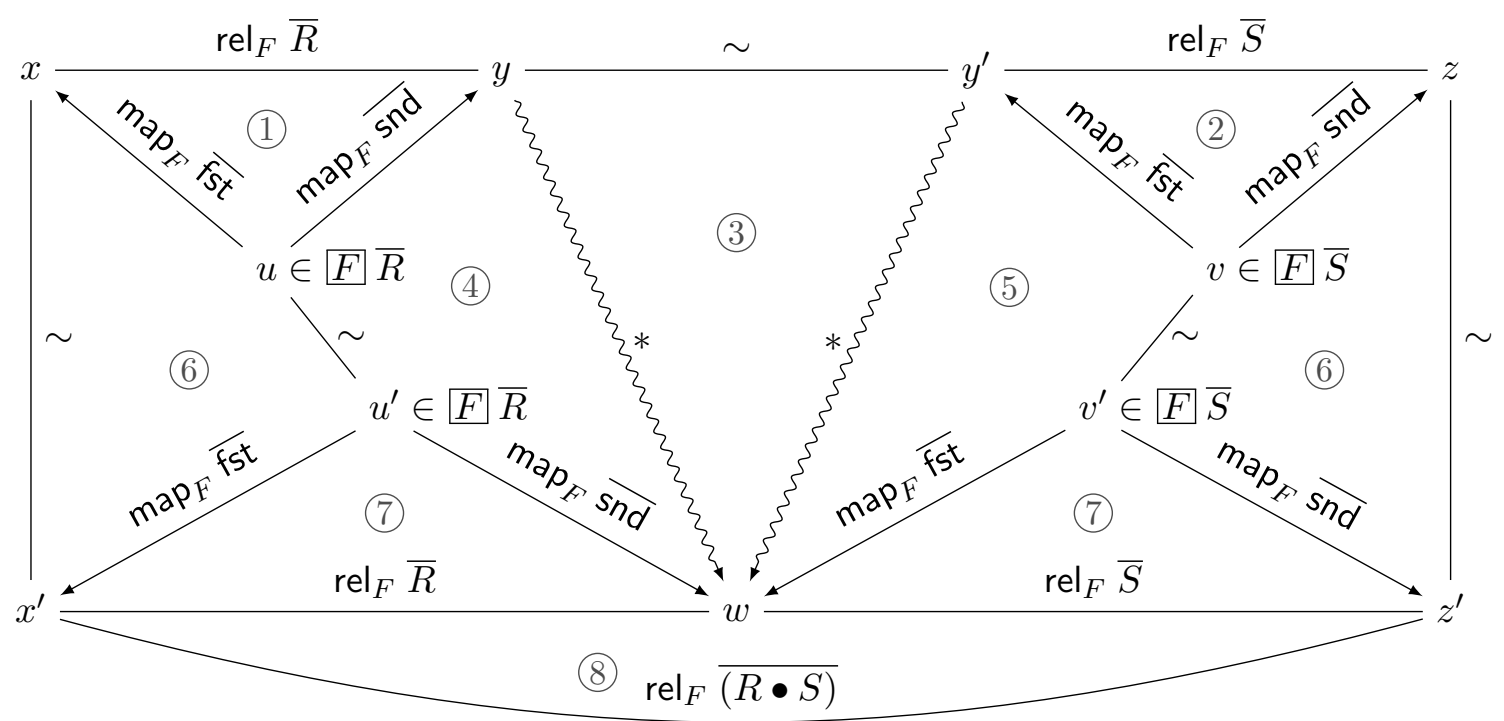

Figure 1: Proof diagram for Theorem 3.21

First (1), by IN_REL, there is a $u \in F \bar{R}$ such that $x=\operatorname{map}_{F} \overline{\text { fst }} u$ and $y=\operatorname{map}_{F} \overline{\text { snd }} u$. Similarly, $\operatorname{rel}_{F} \bar{S} y^{\prime} z$ yields a $v$ with the corresponding properties (2).

Second, by (i), $y \sim y^{\prime}$ implies $y \stackrel{*}{\rightsquigarrow} y^{\prime}$. Since $\rightsquigarrow$ is confluent, there exists a $w$ such that $y \stackrel{*}{\rightsquigarrow} w$ and $y^{\prime} \stackrel{*}{\rightsquigarrow} w$ (3). By induction on $\stackrel{*}{\rightsquigarrow}$ using (ii), $y \stackrel{*}{\rightsquigarrow} w$ factors through the projection $y=\operatorname{map}_{F} \overline{\text { snd }} u$ and we obtain a $u^{\prime}$ such that $u \sim u^{\prime}$ and $w=\operatorname{map}_{F} \overline{\text { snd }} u^{\prime}$ and $\operatorname{set}_{F, i} u^{\prime} \subseteq \operatorname{set}_{F, i} u$ for all $i$ (4). Analogously, we obtain $v^{\prime}$ corresponding to $y^{\prime}$ and $v$ (5). Set

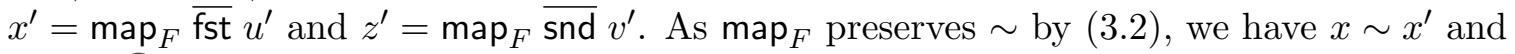
$z \sim z^{\prime}$ (6).

Next, we focus on the two triangles at the bottom (7). By defininition of $F$, $\operatorname{set}_{F, i} u^{\prime} \subseteq$ $\operatorname{set}_{F, i} u$ for all $i$ and $u \in F \bar{R}$ imply $u^{\prime} \in F \bar{R}$; similarly $v^{\prime} \in F \bar{S}$. Now, $u^{\prime}$ and $v^{\prime}$ are the witnesses to the existential in IN_REL for $x^{\prime}$ and $w$, and $w$ and $z^{\prime}$, respectively. So $\left(x^{\prime}, w\right) \in \operatorname{rel}_{F} \bar{R}$ and $\left(w, z^{\prime}\right) \in \operatorname{rel}_{F} \bar{S}$, i.e., $\left(x^{\prime}, z^{\prime}\right) \in\left(\operatorname{rel}_{F} \bar{R} \bullet \operatorname{rel}_{F} \bar{S}\right)$. Finally, as $F$ is a BNF, $\left(x^{\prime}, z^{\prime}\right) \in \operatorname{rel}_{F} \overline{(R \bullet S)}$ follows with subdistributivity REL_COMP (8).

Example 3.22. For distinct lists, we have $\left(\sim_{\text {dlist }}\right)=(\stackrel{*}{\rightsquigarrow}$ dlist $)$ and $\rightsquigarrow$ dlist is confluent. However, condition (ii) of Theorem 3.21 does not hold. For example, for $x=[(1, a),(1, b)]$, we have $\operatorname{map}_{\text {list }}$ fst $x=[1,1] \rightsquigarrow$ dlist $[1]$, and yet there is no $y$ such that $x \sim_{\text {dlist }} y$ and $\operatorname{map}_{\text {list }}$ fst $y=[1]$. The problem is that the projection map list fst makes different atoms of $x$ equal and $\rightsquigarrow$ dist removes equal atoms, but the removal cannot be mimicked on $x$ itself. Fortunately, we can also add equal atoms instead of removing them. Define $\rightsquigarrow^{\prime}$ dlist by

$$
x s \cdot y s \rightsquigarrow^{\prime} \text { dlist } x s \cdot[x] \cdot y s \text { if } x \in \text { set } y s
$$

Then, $\rightsquigarrow^{\prime}$ dlist is confluent and factors through projections. So distinct lists inherit the BNF structure from lists by Theorem 3.21 and either Lemma 3.9 or Lemma 3.11.

Example 3.23. The free monoid over atoms $\alpha$ consists of all finite lists $\alpha$ list. The free idempotent monoid $\alpha$ fim is then the quotient $\alpha$ list $/ \sim_{f i m}$ where $\sim_{f i m}$ is the equivalence closure of the idempotence law for list concatenation

$$
x s \cdot y s \cdot z s \rightsquigarrow \text { fim }_{\mathrm{m}} x s \cdot y s \cdot y s \cdot z s
$$


We have oriented the rule such that it introduces rather than removes the duplication. In term rewriting, the rule is typically oriented in the other direction [Hul80] such that the resulting rewriting system terminates; however, this classical relation $\sim_{\text {fim }}$ is not confluent: $a b a b c b a b c$ has two normal forms $a \underline{b a b c b a b c} \sim \sim_{f i m} \underline{a b a b c} \sim \sim_{f i m} a b c$ and $\underline{a b a b} c b a b c \sim \sim_{f i m} a b c b a b c$ (redexes are underlined). In contrast, our orientation yields a confluent relation $\rightsquigarrow_{\text {fim }}$, although the formal proof requires some effort. The relation also factors through projections. So by Theorem 3.21 and either Lemma 3.9 or Lemma 3.11, the free idempotent monoid $\alpha$ fim is also a BNF.

Example 3.24. A cyclic list is a finite list where the two ends are glued together. Abbot et al. [AAGM04] define the type of cyclic lists as the quotient that identifies lists whose elements have been shifted. Let $\rightsquigarrow$ rotate denote the one-step rotation of a list, i.e.,

$$
[] \rightsquigarrow \text { rotate }[] \quad[x] \cdot x s \rightsquigarrow \text { rotate } x s \cdot[x]
$$

The quotient $\alpha$ cyclist $=\alpha$ list $/ \stackrel{*}{\rightsquigarrow}$ rotate is a BNF as $\rightsquigarrow$ rotate satisfies the conditions of Theorem 3.21 and Lemmas 3.9 and 3.11.

Example 3.25 (Example 1.1 continued). We prove the fact that $\alpha r e_{a c i}$ is a BNF using Theorem 3.21. The confluent rewrite relation $\rightsquigarrow a c i$ that satisfies the conditions of Theorem 3.21 and whose equivalence closure is $\sim_{a c i}$ is defined inductively as follows.

$$
\begin{array}{ll}
\text { Alt }(\text { Alt } r s) t \rightsquigarrow a c i \text { Alt } r \text { (Alt } s t) & \text { Alt } r \text { (Alt } s t) \rightsquigarrow a c i \text { Alt (Alt } r s) t \\
\text { Alt } r s \rightsquigarrow a c i \text { Alt } s r & r \rightsquigarrow a c i \text { Alt } r r \\
r \rightsquigarrow a c i r & \\
r \rightsquigarrow a c i r^{\prime} \Longrightarrow s \rightsquigarrow a c i s^{\prime} \Longrightarrow \text { Alt } r s \rightsquigarrow a c i \text { Alt } r^{\prime} s^{\prime} \\
r \rightsquigarrow a c i r^{\prime} \Longrightarrow s \rightsquigarrow a c i s^{\prime} \Longrightarrow \text { Conc } r s_{a c i} \text { Conc } r^{\prime} s^{\prime} \\
r \rightsquigarrow a c i r^{\prime} \Longrightarrow \text { Star } r \rightsquigarrow a c i \text { Star } r^{\prime}
\end{array}
$$

Example 3.26. We now consider a variant of Example 1.1, where we quotient the regular expressions by $\sim_{a c i d z}$, the least equivalence relation satisfying

$$
\begin{array}{lll}
\text { Alt }(\text { Alt } r s) t \sim_{a c i d z} \text { Alt } r(\text { Alt } s t) & \text { Alt } r s \sim_{a c i d z} \text { Alt } s r & \text { Alt } r r \sim_{a c i d z} r \\
\text { Conc Zero } r \sim_{a c i d z} \text { Zero } & \text { Alt Zero } r \sim_{a c i d z} r & \\
\text { Conc }(\text { Alt } r s) t \sim_{a c i d z} \text { Alt }(\text { Conc } r t) & (\text { Conc } s t) \\
r \sim_{a c i d z} r^{\prime} \Longrightarrow s \sim_{a c i d z} s^{\prime} \Longrightarrow \text { Alt } r s \sim_{a c i d z} \text { Alt } r^{\prime} s^{\prime} \\
r \sim_{a c i d z} r^{\prime} \Longrightarrow \text { Conc } r s \sim_{a c i d z} \text { Conc } r^{\prime} s
\end{array}
$$

This relation is of special interest because, like $\sim_{a c i}$, it gives rise to a finite automaton construction when computing with Brzozowski derivatives of regular expressions equated by $\sim_{a c i d z}$ and, moreover, the constructed automaton is isomorphic to the one obtained by the subset construction via Antimirov's partial derivatives [NT14, Section 4.2].

A first observation is that, unlike $\sim_{a c i}$, the relation $\sim_{a c i d z}$ does not preserve the regular expression's setter: the offending rule being Conc Zero $r \sim_{a c i d z}$ Zero. Nevertheless, $\sim_{a c i d z}$ satisfies (3.2), which together with the fact that regular expressions are finite objects (and thus have finitely many atoms) allows us to use Lemma 3.11 to obtain (3.10).

As before, we aim to apply Theorem 3.21 to show subdistributivity. Notably, the above relation is not a congruence: one is not allowed to apply the equivalence under the Star constructor and in the second argument of Conc. Thus, our confluent "rewrite" relation 
$\rightsquigarrow a c i d z$ also inherits these constraints. Moreover, it uses two auxiliary functions elim_zeros and distribute to handle the new (compared to $\sim_{a c i}$ ) cases related to the Zero and Conc operators.

$$
\begin{aligned}
& \text { elim_zeros (Alt } r s)=\underline{\text { let }} r^{\prime}=\text { elim_zeros } r ; s^{\prime}=\text { elim_zeros } s \underline{\text { in }} \\
& \text { if } r^{\prime}=\text { Zero then } s^{\prime} \text { else if } s^{\prime}=\text { Zero then } r^{\prime} \text { else Alt } r^{\prime} s^{\prime} \\
& \text { elim_zeros (Conc } r s)=\underline{\text { let }} r^{\prime}=\text { elim_zeros } r \text { in if } r^{\prime}=\text { Zero then Zero else Conc } r^{\prime} s \\
& \text { elim_zeros } r=r \\
& \text { distribute } t(\text { Alt } r s)=\text { Alt }(\text { distribute } t r)(\text { distribute } t s) \\
& \text { distribute } t \text { (Conc } r s)=\text { Conc }(\text { distribute } t r) s \\
& \text { distribute } t r=\text { Conc } r t \\
& \text { Alt (Alt } r s) t \rightsquigarrow a c i d z \text { Alt } r \text { (Alt } s t \text { ) } \\
& \text { Alt } \left.r \text { (Alt } s t) \rightsquigarrow_{a c i d z} \text { Alt (Alt } r s\right) t \\
& \text { Alt } r s \rightsquigarrow a c i d z \text { Alt } s r \\
& r \rightsquigarrow \text { acidz Alt } r r
\end{aligned}
$$

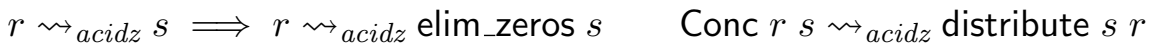

$$
\begin{aligned}
& r \rightsquigarrow a c i d z r \\
& r \rightsquigarrow_{\text {acidz }} r^{\prime} \Longrightarrow s \rightsquigarrow_{\text {acidz }} s^{\prime} \Longrightarrow \text { Alt } r s \rightsquigarrow_{\text {acidz Alt } r^{\prime} s^{\prime}} \\
& r \rightsquigarrow \text { acidz } r^{\prime} \Longrightarrow \text { Conc } r s \rightsquigarrow a c i d z \text { Conc } r^{\prime} s
\end{aligned}
$$

Showing confluence of $\rightsquigarrow a c i d z$ is challenging because $\rightsquigarrow a c i d z$ does not terminate due to $r \rightsquigarrow$ acidz Alt $r$. In fact, we proceed by showing strong confluence. The above rules are carefully designed to be restrictive in what can be rewritten in a single step (fewer restrictions would result in more cases that must be considered) and just permissive enough to be able to join the critical pairs by performing only a single step on one side. In contrast, establishing that $\sim_{a c i d z}$ is the equivalence closure of $\rightsquigarrow_{a c i d z}$ is routine. The missing bit that $\rightsquigarrow_{\text {acidz }}$ factors through projections is also a straightforward induction proof, after showing that the sets of atoms can only decrease along $\rightsquigarrow a c i d z$ (which happens when elim_zeros is used). Altogether, $\rightsquigarrow_{\text {acidz }}$ satisfies the assumption of Theorem 3.21 and allows us to lift the BNF structure of $\alpha$ re to the quotient type:

$$
\text { quotient_type } \alpha r e_{a c i d z}=\alpha \mathrm{re} / \sim_{a c i d z}
$$

We note that the Theorem 3.21's counterpart from our conference paper [FLST20, Theorem 4] does not apply to $\rightsquigarrow a c i d z$ as it required the rewrite relation to preserve the setters.

3.5. Non-emptiness witnesses. An often neglected (also by us in Section 2.1), but important additional piece of information that BNFs carry are the so-called non-emptiness witnesses [BPT15]. These are constants $\mathrm{w}:: \alpha_{i_{1}} \rightarrow \cdots \rightarrow \alpha_{i_{k}} \rightarrow \bar{\alpha} F$, where $I_{\mathrm{w}}=$ $\left\{i_{1}, \ldots, i_{k}\right\} \subseteq\{1, \ldots, n\}$, which capture the information which atoms must be given to construct a value of $\bar{\alpha} F$. The constants are subject to the following property for all $i$ :

$$
a_{i} \in \operatorname{set}_{F, i}\left(\mathrm{w} b_{i_{1}} \cdots b_{i_{k}}\right) \Longrightarrow i \in I_{\mathrm{w}} \wedge a_{i}=b_{i} .
$$

For example, the product type has a single witness Pair :: $\alpha \rightarrow \beta \rightarrow \alpha \times \beta$, whereas the sum type has two: Inl :: $\alpha \rightarrow \alpha+\beta$ and Inr :: $\beta \rightarrow \alpha+\beta$. Non-emptiness witnesses are used to prove that datatypes are non-empty, a requirement for introducing new types in HOL [Pau06]. The fewer arguments a witness has, the more useful it is when proving non-emptiness. Hence, 
a witness with the (index) set of arguments $I$ subsumes another with the set $J$ if $I \subseteq J$. BNFs carry a complete set of witnesses that are minimal with respect to subsumption.

The (co)datatype commands also automatically produce a complete set of witnesses for the resulting types. For example, the list type has a single witness [] :: $\alpha$ list. It forms a complete set because it takes no arguments and thus subsumes every other possible witness. Similarly, the coinductive lists from Example 3.20 have a single witness LNil :: $\alpha$ llist.

Witnesses can be lifted from the raw type to the quotient type because

$$
\operatorname{set}_{Q, i}[x]_{\sim}=\bigcap_{y \in\left[\operatorname{map}_{F} \overline{\mathrm{e}} x\right]_{\sim}}\left\{a \mid \mathfrak{e} a \in \operatorname{set}_{F, i} y\right\} \subseteq \operatorname{set}_{F, i} x
$$

for all $x$. However, the set of witnesses obtained by lifting may stop being complete. For example, lifting from $\alpha$ llist $\times \beta$ to terminated lazy lists results in a single witness tlnil :: $\beta \rightarrow(\alpha, \beta)$ tllist, which corresponds to the witness $\lambda b$. (LNil, $b)$ on the underlying type. Yet there is a second witness tlconst :: $\alpha \rightarrow(\alpha, \beta)$ tllist lifted from $\lambda a$. (Iconst $a$, undef), where the function Iconst is defined corecursively by lconst $a=$ LCons $a$ (Iconst $a$ ) and undef is an unspecified value of type $\beta$. The function $f=(\lambda a$. (Iconst $a$, undef $))$ is not a witness on the raw type because it violates the witness property: $f a$ always contains undef as an atom of type $\beta$, yet $f$ does not take an argument corresponding to that type. In contrast, there is no such atom in tlconst $a$, as we will prove in Example 4.2. Identifying such additional witnesses arising from the equivalence relation's influence on the setters requires manual proofs.

3.6. Partial quotients. So far, we have focused on total quotients generated by an equivalence relation. If the relation $\sim$ is only a partial equivalence relation, i.e., symmetric and transitive but not necessarily reflexive, then the resulting quotient is a partial quotient. Every partial quotient $\bar{\alpha} Q=\bar{\alpha} F / \sim$ factors into a subtype $\bar{\alpha} T$ of $\bar{\alpha} F$ and a total quotient $\bar{\alpha} T / \sim^{\prime}$.

We can therefore combine the conditions for total quotients with those for subtypes. Let Field $\sim$ denote the field of the symmetric relation $\sim$, i.e. Field $\sim=\{x \mid \exists y . x \sim y\}$. Define $\bar{\alpha} T$ as isomorphic to Field $\sim$. The partial equivalence relation $\sim$ on $\bar{\alpha} F$ yields an equivalence relation $\sim^{\prime}$ on $\bar{\alpha} T$. Clearly, $\bar{\alpha} Q$ is isomorphic to $\bar{\alpha} T / \sim^{\prime}$.

The subtype $\bar{\alpha} T$ inherits the BNF structure from $\bar{\alpha} F$ under two conditions [Bie15,LS18]: (1) The set Field $\sim$ must be closed under the mapper $\operatorname{map}_{F}$, i.e., $\operatorname{map}_{F} \bar{f} x \in$ Field $\sim$ for all $\bar{f}$ and $x \in$ Field $_{\sim}$.

(2) Whenever $\operatorname{map}_{F} \overline{\text { fst }} z \in$ Field $\sim$ and $\operatorname{map}_{F} \overline{\text { snd }} z \in$ Field $_{\sim}$, then there exists a $y \in$ Field $_{\sim}$ with $\forall i$. $\operatorname{set}_{F, i} y \subseteq \operatorname{set}_{F, i} z$ and $\operatorname{map}_{F} \overline{\text { fst }} y=\operatorname{map}_{F} \overline{\text { fst }} z$ and $\operatorname{map}_{F} \overline{\operatorname{snd}} y=\operatorname{map}_{F} \overline{\text { snd }} z$.

The first condition follows from $\operatorname{map}_{F}$ preserving $\sim$ by the definition of Field $\sim$. The second condition does not follow from our conditions for total quotients. It ensures that there is a suitable witness $y$ for IN_REL to relate two values in the lifted relation $\operatorname{rel}_{F} \bar{R}$.

Example 3.27. In Example 3.2, the quotient $\alpha F_{\text {seq }} / \sim_{a e}$ of sequences $\alpha F_{\text {seq }}=n a t \rightarrow \alpha$ does not inherit the BNF structure because $\sim_{a e}$ does not satisfy (3.9). We now restrict the equivalence relation to sequences that contain only finitely many different values. That is, the partial equivalence relation $\sim_{f a e}$ relates $x$ to $y$ iff $\{n \mid x n \neq y n\}$ and range $x$ and range $y$ are finite, where range $f=f\langle$ UNIV $\rangle$ denotes the range of $f$. So Field $\sim_{\sim_{a e}}=\{x \mid$ finite $($ range $x)\}$.

By the two-step approach, we first define the functor $\alpha F_{f s e q}$ of finitely-valued sequences as the subtype of $F_{s e q}$. As Field $\sim_{f a e}$ satisfies the above subtype conditions, $F_{f s e q}$ inherits the BNF structure from $F_{\text {seq }}$. Second, we define the functor $F_{\text {fae }}$ of finitely-valued infinitelydifferent sequences as the total quotient of $F_{f s e q}$ over $\sim_{f a e}$. By Theorem 3.19, $F_{f a e}$ inherits the 
BNF structure; wide intersections (3.9) are trivially preserved by Lemma 3.11 as sequences in $F_{f s e q}$ contain only finitely many values.

Inheriting the BNF structure through the subtype $\bar{\alpha} T$ works in many cases. The next example shows that this may fail in pathological cases though. So it might be worthwhile to generalize the constructions from the previous sections directly to partial equivalene relations. This is left as future work.

Example 3.28. Let the partial equivalence relation $\sim$ relate two finite lists $x s$ and $y s$ iff they contain the same elements, i.e., set $x s=$ set $y s$, and each contains at least one element twice. So Field $\sim$ consists of all lists that contain at least one element twice. Let $\alpha T$ be the corresponding subtype of lists. Then $\alpha T$ does not inherit the BNF structure from lists because condition 2 from above does not hold. For the list $z s=[(1, a),(1, b),(2, b)]$, we have map fst $z s=[1,1,2] \in$ Field $_{\sim}$ and map snd $z s=[a, b, b] \in$ Field $_{\sim}$, yet $z s$ is the only list that projects to these two lists and $z s \notin$ Field $_{\sim}$. This failure is not just because condition 2 is too strong; with the mapper for lists, $T$ does not satisfy REL_COMP for the relator defined via IN_REL. Nevertheless, the quotient $T / \sim$ with the inherited mapper becomes the type of non-empty finite sets, which does satisfy the BNF properties.

\section{IMPLEMENTATION}

We provide an Isabelle/HOL command that automatically lifts the BNF structure to total quotient types. The command requires the user to discharge our conditions on the equivalence relation. Upon success, it defines the mapper, setters, the relator, and lifted non-emptiness witnesses, and proves the BNF axioms and transfer rules. The constants' definitions and transfer rules are described in more detail below. Eventually, the command registers the quotient type with the BNF infrastructure for use in future (co)datatype definitions. The command was implemented in 1590 lines of Isabelle/ML. All automated proofs are checked by Isabelle's kernel. Support for partial quotients is left for future work.

4.1. The lift_bnf command. Our implementation extends the interface of the existing lift_bnf command for subtypes [Bie15]. Given a quotient type $\bar{\alpha} Q=\bar{\alpha} F / \sim$,

lift_bnf $\bar{\alpha} Q$ [wits: $\bar{w}$ ]

asks the user to prove the conditions (3.9) and (3.14) of Theorem 3.19, where (3.9) is expressed in terms of (3.10) according to Lemma 3.10. Since the quotient construction already requires that $\sim$ be an equivalence relation, the remaining condition (3.1) holds trivially. The user can provide optional non-emptiness witnesses $\bar{w}$ as functions mapping to the raw type $\bar{\alpha} F$, which adds the corresponding proof obligations (see Section 3.5).

Example 4.1 (Example 3.22 continued). Distinct lists, when viewed as a quotient of lists by the relation $\sim_{\text {dlist }}$, can be registered as a BNF using lift_bnf $\alpha$ dlist. We do not specify additional witnesses because the list witness [] can be lifted and is already as general as possible. Two proof obligations are presented to the user:

$$
\begin{gathered}
\forall R S . R \bullet S \neq\{\} \Longrightarrow \operatorname{rel}_{\text {list }} R \bullet \sim_{\text {dlist }} \bullet \operatorname{rel}_{\text {list }} S \subseteq \sim_{\text {dlist }} \bullet \operatorname{rel}_{\text {list }}(R \bullet S) \bullet \sim_{\text {dlist }} \\
\forall \mathcal{A} . \mathcal{A} \neq\{\} \wedge(\bigcap \mathcal{A}) \neq\{\} \Longrightarrow \bigcap_{A \in \mathcal{A}}[\{x \mid \text { set } x \subseteq A\}]_{\sim_{\text {dlist }}} \subseteq[\{x \mid \text { set } x \subseteq \bigcap \mathcal{A}\}]_{\sim_{\text {dlist }}}
\end{gathered}
$$


The first obligation can be discharged by instantiating Theorem 3.21 as described in Example 3.22. For the second obligation, it suffices to prove that for every list $x$ satisfying

$$
\forall A \in \mathcal{A} . \exists y . x \sim \text { dlist } y \wedge \text { set } y \subseteq A
$$

there exists a list $y^{\prime}$ such that $x \sim$ dlist $y^{\prime}$ and set $y^{\prime} \subseteq \bigcap \mathcal{A}$. We choose $y^{\prime}=x$. Clearly $x \sim_{\text {dlist }} x$, and since $x \sim_{\text {dlist }} y$ implies set $x=$ set $y$, it follows from (4.1) that set $x \subseteq A$ for every $A \in \mathcal{A}$. Alternatively, the preservation of wide intersections follows directly from Lemma 3.11 because set $x$ is finite for every list $x$.

After the conditions have been proved by the user, the command defines the BNF constants. In HOL, the type $\bar{\alpha} Q$ is considered distinct from (but isomorphic to) the set of equivalence classes over $\bar{\alpha} F$. Therefore, the definitions use an abstraction function $\operatorname{abs}_{Q}:: \bar{\alpha} F \rightarrow \bar{\alpha} Q$ and a representation function $\operatorname{rep}_{Q}:: \bar{\alpha} Q \rightarrow \bar{\alpha} F$ to translate between the types. Concretely, we define the quotient's mapper by

$$
\operatorname{map}_{Q} \bar{f}=\operatorname{abs}_{Q} \circ \operatorname{map}_{F} \bar{f} \circ \operatorname{rep}_{Q}
$$

The quotient's setters use the function set ${ }_{1+}$, which maps $\mathfrak{e} a$ to $\{a\}$ and $\circledast$ to \{\} :

$$
\operatorname{set}_{Q, i}=\left(\lambda x \cdot \bigcap_{y \in\left[\operatorname{map}_{F} \overline{\mathfrak{e}} x\right]_{\sim}} \bigcup \operatorname{set}_{1+}\left\langle\operatorname{set}_{F, i} y\right\rangle\right) \circ \operatorname{rep}_{Q}
$$

This definition is equivalent to the characterization in Theorem 3.6. The relator (Theorem 3.16) is lifted similarly. Let $f\langle R\rangle g$ denote the inverse image of the relation $R$ under functions $f$ and $g$, i.e., $(x, y) \in f\langle R\rangle g \longleftrightarrow(f x, f y) \in R$. Then

$$
\operatorname{rel}_{Q} \bar{R}=\operatorname{rep}_{Q}\left\langle\operatorname{map}_{F} \overline{\mathfrak{e}}\left\langle\sim \bullet \operatorname{rel}_{F} \overline{\left(\operatorname{rel}_{1+} R\right)} \bullet \sim\right\rangle \operatorname{map}_{F} \overline{\mathfrak{e}}\right\rangle \operatorname{rep}_{Q}
$$

4.2. Transfer rule generation. The relationship of a quotient's BNF structure to its underlying type allows us to prove additional properties about the former. This is achieved by transfer rules, which drive Isabelle's Transfer tool [HK13] (Section 2.2). Our command automatically proves parametrized transfer rules for the lifted mapper, setters, and relator. Parametrized transfer rules are more powerful because they allow the refinement of nested types [Kun16, Section 4.3]. Such rules involve a parametrized correspondence relation $\operatorname{pcr}_{Q} \bar{A}=\operatorname{rel}_{F} \bar{A} \bullet \mathrm{cr}_{Q}$, where the parameters $\bar{A}$ relate the type arguments of $F$ and $Q$.

The transfer rule of $\operatorname{map}_{Q}$ is unsurprising, as it is the canonical lifting of $\operatorname{map}_{F}$ :

$$
\left(\operatorname{map}_{F}, \operatorname{map}_{Q}\right) \in\left(\overline{(A \mapsto B)} \Leftrightarrow \operatorname{pcr}_{Q} \bar{A} \Leftrightarrow \operatorname{pcr}_{Q} \bar{B}\right)
$$

Setters are not transferred to $\operatorname{set}_{F}$ but to the more complex function from (4.2):

$$
\left(\lambda x . \bigcap_{y \in\left[\operatorname{map}_{F} \overline{\mathfrak{e}} x\right]_{\sim}} \bigcup \operatorname{set}_{1+}\left\langle\operatorname{set}_{F, i} y\right\rangle, \operatorname{set}_{Q, i}\right) \in\left(\operatorname{pcr}_{Q} \bar{A} \mapsto \operatorname{rel}_{s e t} A_{i}\right)
$$

where $(X, Y) \in \operatorname{rel}_{\text {set }} A \longleftrightarrow(\forall x \in X . \exists y \in Y .(x, y) \in A) \wedge(\forall y \in Y . \exists x \in X .(x, y) \in A)$. Similarly, the rule for $Q$ 's relator contains its defining term from (4.3).

Example 4.2 (Example 3.20 continued). Recall that terminated coinductive lists satisfy the conditions for lifting the BNF structure. Thus, we obtain the setter set tllist $, 2_{2}::(\alpha, \beta)$ tllist $\rightarrow$ $\beta$ set among the other BNF operations. We want to prove that $\operatorname{set}_{t l l i s t, 2} x$ is empty for all infinite lists $x$. To make this precise, let the predicate Ifinite $:: \alpha$ llist $\rightarrow$ bool characterize finite coinductive lists. We lift it to $(\alpha, \beta)$ tllist by projecting away the terminator:

$$
\text { lift_definition tlfinite }::(\alpha, \beta) \text { tllist } \rightarrow \text { bool is }(\lambda x \text {. Ifinite }(\text { fst } x))
$$


Therefore, we have to show that $\forall x$. $\neg$ tlfinite $x \Longrightarrow \operatorname{set}_{\text {tllist }, 2} x=\{\}$. Using the transfer rules for the setter and the lifted predicate tlfinite, the transfer proof method reduces the proof obligation to

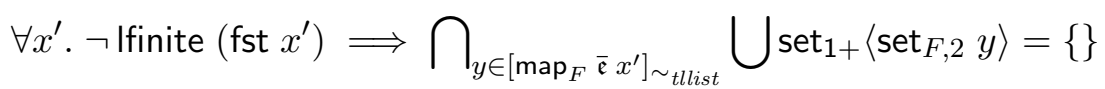

where $x^{\prime}::(\alpha, \beta) F$, and $(\alpha, \beta) F=(\alpha$ llist $\times \beta)$ is the underlying functor of tllist. The rest of the proof, which need not refer to tllist anymore, is automatic. As a corollary of this example, set $_{t l l i s t, 2}$ (tlconst $a$ ) is always empty, a property we used in Section 3.5.

We have also extended lift_bnf to generate transfer rules for subtypes, for which the setters and relator do not change except for the types. For example, if $T$ is a subtype of $F$, $\operatorname{set}_{T, i}$ is transferred to $\operatorname{set}_{F, i}$. Previously no such rules were made available by the command. This limited the properties that could be proved about the setters and relator to those that follow from the generic BNF axioms.

Example 4.3 (Example 3.27 continued). We defined finitely-valued sequences $\alpha F_{f s e q}$ as a subtype of general sequences $\alpha F_{\text {seq. }}$. Our implementation generates the transfer rule

$$
\text { (range, set } \left.f_{s e q}\right) \in\left(\operatorname{pcr}_{f s e q} A \Leftrightarrow \operatorname{rel}_{\text {set }} A\right)
$$

for the setter set $f_{s e q}:: \alpha F_{f s e q} \rightarrow \alpha$ set, where range is the setter of the underlying type $F_{\text {seq }}$.

We use the above rule to show that $F_{f s e q}$ deserves its name, namely that set $f_{s e q} x$ is finite for all $x$. Using that $\operatorname{pcr}_{f s e q}(=)$ is equal to $\operatorname{cr}_{f s e q}$ and rel $_{s e t}(=)$ is the equality relation, the transfer rule implies set $f_{s e q} x=$ range $y$ for all $y$ where $(y, x) \in \operatorname{cr}_{f s e q}$. Recall that the correspondence relation $\mathrm{cr}_{f s e q}$ relates exactly those sequences that have finite range to their isomorphic copies in $F_{f s e q}$. Hence, every $y$ with $(y, x) \in \mathrm{cr}_{f s e q}$ must have finite range. These reasoning steps are automated by the transfer proof method.

\section{RELATED WORK}

Quotient constructions have been formalized and implemented, e.g., in Isabelle/HOL [HK13, KU11, Pau06, Slo97], HOL4 [Hom05], Agda [Vel15, Vel17] (as well as the Cubical Agda variant [VMA21, Vel21]), Cedille [MJS19], Coq [Coh13, CPS03], Lean [ACH19], and Nuprl [Nog02]. None of these works look at the preservation of functor properties except for Avigad et al. [ACH19] (discussed in Section 5.2) and Veltri [Vel17, Vel21].

Veltri [Vel17] studies the special case of when the delay monad is preserved by a quotient of weak bisimilarity, focusing on the challenges that quotients pose in intensional type theory. Furthermore, he [Vel21] examines, using Cubical Agda, how different constructions of the finite powerset functor fset affect codatatype recursion through this functor. He notices that specifying $f$ set as a higher-inductive type via an equational presentation works smoothly in a constructive setting. In contrast, first constructing an intermediate codatatype on the underlying raw type list and then quotienting it with the equivalence relation lifted to the codatatype requires the full axiom of choice for deriving the finality theorem. Analogously, we do not construct an intermediate codatatype and delay the quotienting, but the corecursion directly goes through the quotient BNF thanks to the BNF closure properties.

Abbot et al. [AAGM04] introduce quotient containers as a model of datatypes with permutative structure, such as unordered pairs, cyclic lists, and multisets. The map function of quotient containers does not change the shape of the container. Quotient containers therefore cannot deal with quotients where the equivalence relation takes the identity of 
elements into account, such as distinct lists, finite sets, and the free idempotent monoid. Overall our construction strictly subsumes quotient containers.

5.1. Quotients in the category of Sets. BNFs are accessible functors in the category of Sets. We therefore relate to the literature on when quotients preserve functors and their properties in Set.

Trnková [Trn69] showed that all Set functors preserve non-empty intersections: in our notation $F A \cap F B=F(A \cap B)$ whenever $A \cap B \neq\{\}$. Empty intersections need not be preserved though. Functors that do are called regular [Trn71] or sound [AGT10]. All BNFs are sound as $F A=\left\{x \mid \operatorname{set}_{F} x \subseteq A\right\}$. The naive quotient construction can lead to unsound functors, as shown in Example 3.1.

Every unsound functor can be "repaired" by setting $F\{\}$ to the distinguished points $\mathrm{dp}_{F}$. We write $F^{\prime}$ for the repaired action.

$$
F{ }^{\prime} A= \begin{cases}\mathrm{dp}_{F} & \text { if } A=\{\} \\ F A & \text { otherwise }\end{cases}
$$

Trnková characterizes the distinguished points $\mathrm{dp}_{F}$ as the natural transformations from $C_{1,0}$ to $F$ where $C_{1,0}\{\}=\{\}$ and $C_{1,0} A=\{\circledast\}$ for $A \neq\{\}$. Barr [Bar93] and Gumm [Gum05] use equalizers instead of natural transformations to define the distinguished points of univariate functors:

$$
\mathrm{dp}_{F}=\left\{x \mid \operatorname{map}_{F}\left(\lambda_{-} . \text {True }\right) x=\operatorname{map}_{F}\left(\lambda_{-} . \text {False }\right) x\right\}
$$

The case distinction in (5.1) makes it hard to work with repaired functors, especially as the case distinctions proliferate for multivariate functors. Instead, we repair the unsoundness by avoiding empty sets altogether. Our characterization $F A$ in Lemma 3.5 effectively derives the quotient from $(1+\alpha) F$ instead of $\alpha F$. Moreover, our characterization of $F \bar{A}$ generalizes Barr and Gumm's definition of distinguished points: for $\bar{A}=\{\},(3.5)$ simplifies to (5.2), using preimage preservation (3.7). The resulting quotient is the same because $[F \bar{A}]_{\sim}=[F \bar{A}]_{\sim}$ if $A_{i} \neq\{\}$ for all $i$.

Given the other BNF properties, subdistributivity is equivalent to the functor preserving weak pullbacks. Adámek et al. [AGT10] showed that an accessible Set functor preserves weak pullbacks iff it has a so-called dominated presentation in terms of flat equations $E$ over a signature $\Sigma$. This characterization does not immediately help with proving subdistributivity, though. For example, the finite set quotient $\alpha$ fset $=\alpha$ list $/ \sim_{\text {fset }}$ comes with the signature $\Sigma=\left\{\sigma_{n} \mid n \in \mathbb{N}\right\}$ and the equations $\sigma_{n}\left(x_{1}, \ldots x_{n}\right)=\sigma_{m}\left(y_{1}, \ldots, y_{m}\right)$ whenever $\left\{x_{1}, \ldots, x_{n}\right\}=\left\{y_{1}, \ldots, y_{m}\right\}$. Proving domination for this presentation boils down to proving subdistributivity directly. Our criterion using a confluent relation (Theorem 3.21) is only sufficient, not necessary, but it greatly reduces the actual proof effort.

5.2. Lean's quotients of polynomial functors. Avigad et al. [ACH19] proposed quotients of polynomial functors (QPF) as a model for datatypes. QPFs generalize BNFs in that they require less structure: there is no setter and the relator need not satisfy subdistributivity. Nevertheless, the quotient construction is similar to ours. Without loss of generality, we consider in our comparison only the univariate case $\alpha Q=\alpha F / \sim$. 
The main difference lies in the definition of the liftings lift $F$ of predicates $P:: \alpha \rightarrow$ bool and relations $R:: \alpha \otimes \beta$. In our notation, $\operatorname{lift}_{F} P$ corresponds to $\lambda x . x \in F\{a \mid P a\}$ and $\operatorname{lift}_{F} R$ to rel $_{F} R$. QPFs define these liftings for the quotient $Q$ as follows:

$$
\operatorname{lift}_{Q} P[x]_{\sim}=\left(\exists x^{\prime} \in[x]_{\sim} . P x^{\prime}\right) \quad \operatorname{lift}_{Q} R[x]_{\sim}[y]_{\sim}=\left(\exists x^{\prime} \in[x]_{\sim} . \exists y^{\prime} \in[y]_{\sim} \cdot R x^{\prime} y^{\prime}\right)
$$

That is, these definitions correspond to the naive construction $Q A=\left[\begin{array}{ll}F & A]_{\sim} \text { and }\end{array}\right.$ $\operatorname{rel}_{Q} R=\left[\operatorname{rel}_{F} R\right]_{\sim}$, where $[(x, y)]_{\sim}=\left([x]_{\sim},[y]_{\sim}\right)$. As discussed above, the resulting quotient may be an unsound functor. Consequently, lifting of predicates does not preserve empty intersections in general. This hinders modular proofs. For example, suppose that a user has already shown lift $Q P_{1} x$ and lift $P_{Q} x$ for some value $x$ and two properties $P_{1}$ and $P_{2}$. Then, to deduce lift $F\left(\lambda a . P_{1} a \wedge P_{2} a\right) x$, they would have to prove that the two properties do not contradict each other, i.e., $\exists a . P_{1} a \wedge P_{2} a$. Obviously, this makes modular proofs harder as extra work is needed to combine properties.

QPFs use $\operatorname{lift}_{F} P$ in the induction theorem for datatypes. So when a datatype recurses through tllist, the aforementioned obstacle spreads to proofs by induction: splitting a complicated inductive statement into smaller lemmas is not for free. Moreover, lift $Q$ holds for fewer values, as the next example shows. Analogous problems arise in QPFs for relation lifting, which appears in the coinduction theorem.

Example 5.1 (Example 4.2 cont.). Consider the infinite repetition tlconst $a::(\alpha, \beta)$ tllist of the atom $a$ as a terminated lazy list. As tlconst $a$ contains only as, one would expect that lift $_{\text {tllist }}\left(\lambda a^{\prime} . a^{\prime}=a\right)\left(\lambda_{-}\right.$. False) (tlconst $\left.a\right)$ holds. Yet this property is provably false.

These issues would go away if lift ${ }_{Q}$ was defined following our approach for $Q A=[E A]_{\sim}$ and rel $_{Q}$ as in Theorem 3.16. These definitions do not rely on the additional BNF structure; only $\operatorname{map}_{Q}$ is needed and QPFs define $\operatorname{map}_{Q}$ like we do. The repair should therefore work for the general QPF case as well.

\section{Conclusion}

We have described a sufficient criterion for quotient types to be able to inherit the BNF structure from the underlying type. We have demonstrated the effectiveness of the criterion by automating the BNF "inheritance" in the form of the lift_bnf command in Isabelle/HOL and used it (which amounts to proving the criterion) for several realistic quotient types. We have also argued that our treatment of the quotient's setter and relator to avoid unsoundness carries over to more general structures, such as Lean's QPFs.

As future work, we plan to investigate quotients of existing generalizations of BNFs to coand contravariant functors [LS18] and functors operating on small-support endomorphisms and bijections [BGPT19]. Furthermore, we would like to provide better automation for proving subdistributivity via confluent rewrite systems as part of lift_bnf.

\section{ACKNOWLEDGMENT}

We thank David Basin for supporting this work, Ralf Sasse and Andrei Popescu for insightful discussions about confluent relations, BNFs, their preservation of wide intersections, and ways to express the setters in terms of the mapper, and Jasmin Blanchette and the anonymous IJCAR and LMCS reviewers for numerous comments on earlier drafts of this article, which 
helped to improve the presentation. Julian Biendarra developed the original lift_bnf command for subtypes, which we extended to quotient types in this work.

\section{REFERENCES}

[AAGM04] Michael Abbott, Thorsten Altenkirch, Neil Ghani, and Conor McBride. Constructing polymorphic programs with quotient types. In Dexter Kozen, editor, MPC 2004, volume 3125 of $L N C S$, pages 2-15, Berlin, Heidelberg, 2004. Springer.

[ACH19] Jeremy Avigad, Mario Carneiro, and Simon Hudon. Data types as quotients of polynomial functors. In John Harrison, John O'Leary, and Andrew Tolmach, editors, ITP 2019, volume 141 of LIPIcs, pages 6:1-6:19. Schloss Dagstuhl - Leibniz-Zentrum für Informatik, 2019. doi: 10.4230/LIPIcs. ITP. 2019.6.

[AGT10] Jirí Adámek, H. Peter Gumm, and Vera Trnková. Presentation of set functors: A coalgebraic perspective. J. Log. Comput., 20(5):991-1015, 2010. doi:10.1093/logcom/exn090.

[Bar93] Michael Barr. Terminal coalgebras in well-founded set theory. Theor. Comput. Sci., 114(2):299315, 1993. doi:10.1016/0304-3975(93)90076-6.

[BGPT19] Jasmin Christian Blanchette, Lorenzo Gheri, Andrei Popescu, and Dmitriy Traytel. Bindings as bounded natural functors. PACMPL, 3(POPL):22:1-22:34, 2019. doi:10.1145/3290335.

$\left[\mathrm{BHL}^{+} 14\right]$ Jasmin Christian Blanchette, Johannes Hölzl, Andreas Lochbihler, Lorenz Panny, Andrei Popescu, and Dmitriy Traytel. Truly modular (co)datatypes for Isabelle/HOL. In Gerwin Klein and Ruben Gamboa, editors, ITP 2014, volume 8558 of LNCS, pages 93-110. Springer, 2014.

[Bie15] Julian Biendarra. Functor-preserving type definitions in Isabelle/HOL. Bachelor thesis, Fakultät für Informatik, Technische Universität München, 2015.

[BKT17] David A. Basin, Srdan Krstic, and Dmitriy Traytel. Almost event-rate independent monitoring of metric dynamic logic. In Shuvendu K. Lahiri and Giles Reger, editors, RV 2017, volume 10548 of $L N C S$, pages 85-102. Springer, 2017. doi:10.1007/978-3-319-67531-2_6.

[BLS20] David A. Basin, Andreas Lochbihler, and S. Reza Sefidgar. CryptHOL: Game-based proofs in higher-order logic. J. Cryptology, 33:494-566, 2020. doi:10.1007/s00145-019-09341-z.

[BPT14] Jasmin Christian Blanchette, Andrei Popescu, and Dmitriy Traytel. Cardinals in Isabelle/HOL. In Gerwin Klein and Ruben Gamboa, editors, ITP 2014, volume 8558 of LNCS, pages 111-127. Springer, 2014. doi:10.1007/978-3-319-08970-6_8.

[BPT15] Jasmin Christian Blanchette, Andrei Popescu, and Dmitriy Traytel. Witnessing (co)datatypes. In Jan Vitek, editor, ESOP 2015, volume 9032 of LNCS, pages 359-382. Springer, 2015. doi: 10.1007/978-3-662-46669-8_15.

[CDM13] Cyril Cohen, Maxime Dénès, and Anders Mörtberg. Refinements for free! In Georges Gonthier and Michael Norrish, editors, CPP 2013, volume 8307 of LNCS, pages 147-162. Springer, 2013.

[Coh13] Cyril Cohen. Pragmatic quotient types in Coq. In Sandrine Blazy, Christine Paulin-Mohring, and David Pichardie, editors, ITP 2013, volume 7889 of LNCS, pages 213-228. Springer, 2013. doi: $10.1007 / 978-3-642-39634-2 \_17$.

[CPS03] Laurent Chicli, Loï Pottier, and Carlos Simpson. Mathematical quotients and quotient types in Coq. In Herman Geuvers and Freek Wiedijk, editors, TYPES 2003, volume 2646 of LNCS, pages 95-107. Springer, 2003. doi:10.1007/3-540-39185-1_6.

[DV13] Giuseppe De Giacomo and Moshe Y. Vardi. Linear temporal logic and linear dynamic logic on finite traces. In Francesca Rossi, editor, IJCAI 2013, pages 854-860. IJCAI/AAAI, 2013.

[FLST20] Basil Fürer, Andreas Lochbihler, Joshua Schneider, and Dmitriy Traytel. Quotients of bounded natural functors. In Nicolas Peltier and Viorica Sofronie-Stokkermans, editors, IJCAR 2020, volume 12167 of $L N C S$, pages 58-78. Springer, 2020. doi:10.1007/978-3-030-51054-1_4.

[GS05] H. Peter Gumm and Tobias Schröder. Types and coalgebraic structure. Algebra universalis, 53(2):229-252, 2005. doi:10.1007/s00012-005-1888-2.

[Gum05] H. Peter Gumm. From T-coalgebras to filter structures and transition systems. In José Luiz Fiadeiro, Neil Harman, Markus Roggenbach, and Jan Rutten, editors, Algebra and Coalgebra in Computer Science, volume 3629 of LNCS, pages 194-212, Berlin, Heidelberg, 2005. Springer.

[HK13] Brian Huffman and Ondřej Kunčar. Lifting and Transfer: A modular design for quotients in Isabelle/HOL. In Georges Gonthier and Michael Norrish, editors, CPP 2013, volume 8307 of LNCS, pages 131-146. Springer, 2013. doi:10.1007/978-3-319-03545-1_9. 
[Hom05] Peter V. Homeier. A design structure for higher order quotients. In Joe Hurd and Tom Melham, editors, TPHOLs 2005, volume 3603 of LNCS, pages 130-146, Berlin, Heidelberg, 2005. Springer. doi:10.1007/11541868_9.

[Hul80] Jean-Marie Hullot. A catalogue of canonical term rewrite systems. Technical Report CSL-113, SRI International, 1980.

[KP19] Ondřej Kunčar and Andrei Popescu. From types to sets by local type definition in higher-order logic. J. Autom. Reasoning, 62(2):237-260, 2019. doi:10.1007/s10817-018-9464-6.

[KU11] Cezary Kaliszyk and Christian Urban. Quotients revisited for Isabelle/HOL. In William C. Chu, W. Eric Wong, Mathew J. Palakal, and Chih-Cheng Hung, editors, SAC 2011, pages 1639-1644. ACM, 2011. doi:10.1145/1982185.1982529.

[Kun16] Ondřej Kunčar. Types, Abstraction and Parametric Polymorphism in Higher-Order Logic. PhD thesis, Technical University Munich, Germany, 2016.

[LL19] Peter Lammich and Andreas Lochbihler. Automatic refinement to efficient data structures: A comparison of two approaches. J. Autom. Reasoning, 63(1):53-94, 2019. doi:10.1007/ s10817-018-9461-9.

[Loc10] Andreas Lochbihler. Coinductive. Archive of Formal Proofs, 2010. http://isa-afp.org/entries/ Coinductive.html, Formal proof development.

[Loc19] Andreas Lochbihler. Effect polymorphism in higher-order logic (proof pearl). J. Autom. Reasoning, 63(2):439-462, 2019. doi:10.1007/s10817-018-9476-2.

[LS16] Andreas Lochbihler and Joshua Schneider. Equational reasoning with applicative functors. In Jasmin Christian Blanchette and Stephan Merz, editors, ITP 2016, volume 9807 of LNCS, pages 252-273. Springer, 2016. doi:10.1007/978-3-319-43144-4_16.

[LS18] Andreas Lochbihler and Joshua Schneider. Relational parametricity and quotient preservation for modular (co)datatypes. In Jeremy Avigad and Assia Mahboubi, editors, ITP 2018, volume 10895 of $L N C S$, pages 411-431. Springer, 2018. doi:10.1007/978-3-319-94821-8_24.

[LSBM19] Andreas Lochbihler, S. Reza Sefidgar, David A. Basin, and Ueli Maurer. Formalizing constructive cryptography using CryptHOL. In CSF 2019, pages 152-166. IEEE, 2019. doi:10.1109/CSF. 2019.00018.

[MJS19] Andrew Marmaduke, Christopher Jenkins, and Aaron Stump. Quotient types by normalization in Cedille. In TFP 2019, 2019.

[NK14] Tobias Nipkow and Gerwin Klein. Concrete Semantics - With Isabelle/HOL. Springer, 2014. doi : 10.1007/978-3-319-10542-0.

[Nog02] Aleksey Nogin. Quotient types: A modular approach. In Victor A. Carreño, César A. Muñoz, and Sofiène Tahar, editors, TPHOLs 2002, volume 2410 of LNCS, pages 263-280. Springer, 2002. doi:10.1007/3-540-45685-6_18.

[NT14] Tobias Nipkow and Dmitriy Traytel. Unified decision procedures for regular expression equivalence. In Gerwin Klein and Ruben Gamboa, editors, ITP 2014, volume 8558 of LNCS, pages 450-466. Springer, 2014. doi:10.1007/978-3-319-08970-6_29.

[Pau06] Lawrence C. Paulson. Defining functions on equivalence classes. ACM Trans. Comput. Logic, 7(4):658-675, 2006. doi:10.1145/1183278.1183280.

[Slo97] Oscar Slotosch. Higher order quotients and their implementation in Isabelle/HOL. In Elsa L. Gunter and Amy Felty, editors, TPHOLs 1997, volume 1275 of LNCS, pages 291-306, Berlin, Heidelberg, 1997. Springer. doi:10.1007/BFb0028401.

[Soz10] Matthieu Sozeau. A new look at generalized rewriting in type theory. J. Formalized Reasoning, 2(1):41-62, 2010. doi:10.6092/issn.1972-5787/1574.

[TPB12] Dmitriy Traytel, Andrei Popescu, and Jasmin Christian Blanchette. Foundational, compositional (co)datatypes for higher-order logic: Category theory applied to theorem proving. In LICS 2012, pages 596-605. IEEE Computer Society, 2012. doi:10.1109/LICS.2012.75.

[Trn69] Věra Trnková. Some properties of set functors. Commentationes Mathematicae Universitatis Carolinae, 10(2):323-352, 1969.

[Trn71] Vera Trnková. On descriptive classification of set-functors I. Commentationes Mathematicae Universitatis Carolinae, 12(1):143-174, 1971.

[Vel15] Niccolò Veltri. Two set-based implementations of quotients in type theory. In J. Nummenmaa, O. Sievi-Korte, and E. Mäkinen, editors, SPLST 2015, volume 1525 of CEUR Workshop Proceedings, pages 194-205, 2015. 
[Vel17] Niccolò Veltri. A Type-Theoretical Study of Nontermination. PhD thesis, Tallinn University of Technology, 2017.

[Vel21] Niccolò Veltri. Type-theoretic constructions of the final coalgebra of the finite powerset functor. In Naoki Kobayashi, editor, FSCD 2021, volume 195 of LIPIcs, pages 22:1-22:18. Schloss Dagstuhl Leibniz-Zentrum für Informatik, 2021. doi:10.4230/LIPIcs.FSCD.2021.22.

[VMA21] Andrea Vezzosi, Anders Mörtberg, and Andreas Abel. Cubical agda: A dependently typed programming language with univalence and higher inductive types. J. Funct. Program., 31:e8, 2021. doi:10.1017/S0956796821000034. 